\title{
Next-to-leading-logarithmic power corrections for $N$-jettiness subtraction in color-singlet production
}

\author{
Radja Boughezal \\ High Energy Physics Division, Argonne National Laboratory, Argonne, Illinois 60439, USA \\ Andrea Isgrò \\ Department of Physics and Astronomy, Northwestern University, Evanston, Illinois 60208, USA \\ Frank Petriello \\ High Energy Physics Division, Argonne National Laboratory, Argonne, Illinois 60439, USA \\ and Department of Physics and Astronomy, Northwestern University, Evanston, Illinois 60208, USA
}

(Received 16 February 2018; published 11 April 2018)

\begin{abstract}
We present a detailed derivation of the power corrections to the factorization theorem for the 0 -jettiness event shape variable $\mathcal{T}$. Our calculation is performed directly in QCD without using the formalism of effective field theory. We analytically calculate the next-to-leading logarithmic power corrections for small $\mathcal{T}$ at next-to-leading order in the strong coupling constant, extending previous computations which obtained only the leading-logarithmic power corrections. We address a discrepancy in the literature between results for the leading-logarithmic power corrections to a particular definition of 0 -jettiness. We present a numerical study of the power corrections in the context of their application to the $N$-jettiness subtraction method for higher-order calculations, using gluon-fusion Higgs production as an example. The inclusion of the next-to-leading-logarithmic power corrections further improves the numerical efficiency of the approach beyond the improvement obtained from the leading-logarithmic power corrections.
\end{abstract}

DOI: 10.1103/PhysRevD.97.076006

\section{INTRODUCTION}

The increasing precision of data from the Large Hadron Collider (LHC) and the anticipated precision of potential future experiments require calculations to increasingly high orders in the perturbative expansion of QCD. The calculation of higher-order corrections is complicated by the fact that the real-emission and virtual contributions to the cross section exhibit infrared singularities that cancel only after they are combined. Currently, the fully differential predictions needed to model the final-state cuts imposed in all experimental analyses can be obtained at next-to-next-toleading order (NNLO) in the strong coupling constant for $2 \rightarrow 2$ scattering processes. These have become available only recently due to rapid progress in developing new schemes for the efficient cancellation of infrared singularities [1-8]. With these advances, a new standard in the comparison of theoretical predictions with data from the LHC has been achieved.

Published by the American Physical Society under the terms of the Creative Commons Attribution 4.0 International license. Further distribution of this work must maintain attribution to the author(s) and the published article's title, journal citation, and DOI. Funded by SCOAP ${ }^{3}$.
We discuss here one of these successful new approaches to higher-order QCD calculations, the $N$-jettiness subtraction scheme $[7,8]$. This method uses the $N$-jettiness event shape variable $\mathcal{T}_{N}$ [9] as a resolution parameter to isolate and cancel the double-unresolved singular limits, where two partons become soft and/or collinear, that complicate the calculation of NNLO cross sections. It has led to some of the first calculations for vector boson production in association with a jet [7,10-13] and Higgs production in association with a jet [14] at the LHC through NNLO. It has also led to first predictions for inclusive jet production at NNLO in electronnucleon collisions [15]. The $N$-jettiness subtraction scheme relies upon the introduction of a cutoff $\mathcal{T}_{N}^{\text {cut }}$ that separates the $N+1$ jet region of an $N$-jet production process from the doubly-unresolved limit. The below-cut region is expanded in $\mathcal{T}_{N}^{\text {cut }} / Q$, where $Q$ denotes the hard momentum transfer in the process, in order to allow for an effective field theory calculation using soft-collinear effective theory (SCET) [1620]. A small cutoff is needed so that the power corrections in $\mathcal{T}_{N}^{\text {cut }} / Q$ are negligible. However, the below-cut and abovecut contributions separately depend on logarithms of $\mathcal{T}_{N}^{\text {cut }} / Q$ that only cancel after combining the two regions. For small $\mathcal{T}_{N}^{\text {cut }}$ these logarithms introduce numerical noise that must be controlled. Although the numerics can already be controlled sufficiently for phenomenological applications, it is desirable 
for computational efficiency to reduce the sensitivity of the method to the power corrections. One approach is to analytically calculate the power corrections to the SCET factorization theorem used in the below-cut region. The study of the structure of subleading power corrections to effectivetheory factorization theorems is also of general interest, and has received significant attention recently [21-29].

The importance of and interest in the $N$-jettiness subtraction scheme has motivated several calculations of the power corrections to the factorization theorem for the simplest case of color-singlet production, which uses the 0 -jettiness event shape variable. The leading-logarithmic power corrections to the below-cut Drell-Yan cross section, which scale as $\alpha_{s} \mathcal{T}_{N}^{\text {cut }} \log \mathcal{T}_{N}^{\text {cut }}$ and $\alpha_{s}^{2} \mathcal{T}_{N}^{\text {cut }} \log ^{3} \mathcal{T}_{N}^{\text {cut }}$ at NLO and NNLO respectively, were derived using an effective field theory approach in Ref. [30]. The leading-logarithmic power corrections for both Drell-Yan and gluon-fusion Higgs production were derived using both an effectivetheory method and a direct QCD calculation in Ref. [31]. The corrections for gluon-fusion Higgs production were subsequently considered using an effective-theory approach in Ref. [32]. Particular attention was paid in this work to comparing two different variants of $\mathcal{T}_{0}$, denoted as the leptonic and hadronic definitions. We explicitly define these versions of 0 -jettiness in the next section. Furthermore, a disagreement between the results of Refs. [31] and [30,32] for the hadronic $\mathcal{T}_{0}$ was discussed in this paper.

Motivated both by this discrepancy and by the increasing interest in understanding power corrections in effective field theory, we revisit and extend the calculation of the power corrections to the 0 -jettiness factorization theorem. We attempt to fulfill several goals in this work.

(i) We present a detailed derivation of the leading-power cross section and the subleading power corrections. For the leading-power terms we show explicitly how the calculation using the SCET factorization theorem maps onto a direct derivation using QCD. We study gluon-fusion Higgs production as a representative example. Our aim is to provide a detailed, pedagogical discussion to allow interested readers with differing expertise to follow the derivations in detail.

(ii) We address the discrepancy in the leading-logarithmic (LL) power corrections for the hadronic $\mathcal{T}_{0}$ in Refs. [30,32,31]. We present in detail an explicit calculation of the NLO power corrections for gluonfusion Higgs production directly in QCD, assuming an arbitrary hardness measure used to define $\mathcal{T}_{0}$, making our derivation valid for both the leptonic and hadronic definitions. Our result agrees with the leptonic $\mathcal{T}_{0}$ of Refs. [30,32], but with the hadronic definition in Ref. [31]. We identify a subtlety in the derivation of the power corrections for hadronic $\mathcal{T}_{0}$ that we believe may have been missed in Refs. [30,32], and which can explain the difference between the results in the literature. (iii) In addition to rederiving in detail the leading-logarithmic subleading power corrections, we also derive analytically for the first time the next-to-leadinglogarithmic (NLL) $\mathcal{O}\left(\mathcal{T}_{0}\right)$ power corrections.

(iv) We study in detail the numerical impact of the LL and NLL power corrections for both the hadronic and leptonic $\mathcal{T}$ definitions on the NLO gluon-fusion Higgs cross section computed using $N$-jettiness subtraction. The inclusion of the full power corrections further improves the numerical efficiency of the method beyond the leading-logarithmic improvements observed in previous work [30-32]. Deviations from the NLO correction obtained using dipole subtraction remain below $1 \%$, and at the fewpercent level in the forward rapidity region, for the hadronic $\mathcal{T}$ definition even up to $\mathcal{T}^{\text {cut }}=1 \mathrm{GeV}$. The leptonic definition is nearly identical to the dipole subtraction result for all $\mathcal{T}^{\text {cut }}$ after the inclusion of the NLL power corrections.

Our manuscript is organized as follows. We present our notation and define the versions of 0 -jettiness we consider in Sec. II. For pedagocial purposes, and as a check on our direct derivation of the $\mathcal{T}$ distribution including power corrections, we derive the leading-power result using the SCET factorization theorem in Sec. III. Our direct QCD derivation of the leading-power result, as well as the full $\mathcal{O}(\mathcal{T})$ power corrections, is shown in detail in Sec. IV. We also demonstrate in this section how we believe the discrepancy between Refs. [31] and [30,32] for the hadronic $\mathcal{T}$ case arises. We present numerical results, including evidence for the correctness of the leading-logarithmic power corrections of Ref. [31] for the hadronic $\mathcal{T}$ definition, in Sec. V. Finally, we conclude in Sec. VI. In the Appendix, we list the formulas for the NLL power corrections for the $q g$ and $q \bar{q}$ contributions to Higgs production. The expressions for the dominant $g g$ channel are given in the main text.

\section{NOTATION AND $\mathcal{T}$ DEFINITIONS}

In this section, we present the notation and conventions used in this paper, and define the $\mathrm{N}$-jettiness event-shape variable. To simplify comparison with other results in the literature we adopt whenever possible the notation of Ref. [32].

The most general definition of the $N$-jettiness eventshape variable is

$$
\mathcal{T}_{N}=\sum_{k} \min _{i}\left\{\frac{2 q_{i} \cdot p_{k}}{Q_{i}}\right\}
$$

where the index $i$ runs over the two beam directions and over the $N$ final-state jets. The index $k$ runs over all finalstate partons. The $Q_{i}$ are arbitrary hardness measures that lead to different definitions of $N$-jettiness. For our gluonfusion Higgs production example we will be interested in 0 -jettiness in the situation where there is only a single 
parton in the final state. In this case the relevant event shape variable is

$$
\mathcal{T}_{0}=\min \left\{\frac{2 q_{a} \cdot p_{3}}{Q_{a}}, \frac{2 q_{b} \cdot p_{3}}{Q_{b}}\right\},
$$

where $p_{3}$ is the momentum of the emitted final-state parton. The two beam momenta $q_{a}$ and $q_{b}$ are

$$
q_{a}=\frac{x_{a} \sqrt{s}}{2} n^{\mu}, \quad q_{b}=\frac{x_{b} \sqrt{s}}{2} \bar{n}^{\mu},
$$

where $x_{a}$ and $x_{b}$ are the momentum fractions carried by the two partons, and

$$
n^{\mu} \equiv\left(\begin{array}{l}
1 \\
0 \\
0 \\
1
\end{array}\right), \quad \bar{n}^{\mu} \equiv\left(\begin{array}{c}
1 \\
0 \\
0 \\
-1
\end{array}\right) .
$$

We will use the variables $x_{a}$ and $x_{b}$ to refer to the Born-level momentum fractions, and not for the incoming momentum fractions in the NLO result. This distinction will be important when studying the factorization of the phase space into a Born-level piece and a radiation phase space in Sec. IV. As we will be considering gluon-fusion Higgs production as a pedogogical example, we will have $Q=m_{H}$. From now on the subscript 0 for jettiness will be implicit.

The hardness measures $Q_{a}$ and $Q_{b}$ in Eq. (2) are arbitrary. The leading-power cross section is not affected by this choice, but the subleading power corrections do depend on these normalization factors. While our derivation will be valid for arbitrary $Q_{i}$, we will call particular attention to two possible choices: leptonic $\mathcal{T}$ and hadronic $\mathcal{T}$, following the terminology of Ref. [32]. Leptonic $\mathcal{T}$ is defined by

$$
\text { Leptonic: } \begin{aligned}
Q_{a} & =Q_{b}=Q, \\
\mathcal{T}_{\text {lep }} & =\min \left\{\frac{x_{a} \sqrt{s}}{Q} n \cdot p_{3}, \frac{x_{b} \sqrt{s}}{Q} \bar{n} \cdot p_{3}\right\},
\end{aligned}
$$

while hadronic $\mathcal{T}$ is defined by

$$
\begin{aligned}
\text { Hadronic: } Q_{a} & =x_{a} \sqrt{s}, \quad Q_{b}=x_{b} \sqrt{s}, \\
\mathcal{T}_{\text {had }} & =\min \left\{n \cdot p_{3}, \bar{n} \cdot p_{3}\right\} .
\end{aligned}
$$

\section{LEADING-POWER DERIVATION USING SCET}

As a check on our direct QCD derivation of the 0-jettiness power corrections in Sec. IV, we will compare the leadingpower expression obtained there with the result from the SCET factorization theorem $[9,33]$. According to this result the differential cross section in $\mathcal{T}$ to leading order in the $\mathcal{T} / Q$ expansion can be expressed in terms of universal objects in the effective theory known as hard, soft and beam functions that respectively describe hard radiation, soft radiation, and radiation collinear to a beam direction. We can write the leading-power (LP) result as $[9,33]$

$$
\begin{aligned}
\frac{\mathrm{d} \sigma^{\mathrm{LP}}}{\mathrm{d} \mathcal{T}}= & \int \mathrm{d} x_{a} \int \mathrm{d} x_{b} \int \mathrm{d} \Phi_{H} \int \mathrm{d} \mathcal{T}_{a} \mathrm{~d} \mathcal{T}_{b} \delta\left(\mathcal{T}-\mathcal{T}_{a}-\mathcal{T}_{b}\right) \\
& \times \int \mathrm{d} t_{a} \mathrm{~d} t_{b} B_{a}\left(t_{a}, x_{a}, \mu\right) B_{b}\left(t_{b}, x_{b}, \mu\right) \\
& \times S\left(\mathcal{T}_{a}-\frac{t_{a}}{Q_{a}}, \mathcal{T}_{b}-\frac{t_{b}}{Q_{b}}, \mu\right) H\left(p_{H}, \mu\right)
\end{aligned}
$$

Here, $\Phi_{H}$ denotes the Born phase space for Higgs production, $x_{a}$ and $x_{b}$ denote the Bjorken- $x$ variables for each beam, and $H, B$, and $S$ respectively denote the hard, beam and soft functions. The variables $t_{a}$ and $t_{b}$ parametrize the contributions of the beam sectors to the total 0 -jettiness $\mathcal{T}$. For gluon-fusion Higgs production the Born-level phase space takes the form

$$
\begin{aligned}
\int \mathrm{d} \Phi_{H} & =\frac{(2 \pi)}{2 s x_{a} x_{b}} \int \mathrm{d}^{d} p_{H} \delta\left(p_{H}^{2}-m_{H}^{2}\right) \delta^{(d)}\left(q_{a}+q_{b}-p_{H}\right) \\
& =\frac{(2 \pi)}{2 s x_{a} x_{b}} \delta\left(s x_{a} x_{b}-m_{H}^{2}\right)
\end{aligned}
$$

The factorization formula of Eq. (7) is true to all orders in the strong coupling constant $\alpha_{s}$. In our study we are interested in the leading-power result at NLO, so we can expand each function to the NLO level to obtain the fixedorder result for the differential cross section. Using a convolution symbol to abbreviate the integrals appearing in Eq. (7), we find the following four convolutions contributing to the NLO cross section:

$$
\begin{aligned}
\frac{\mathrm{d} \sigma_{\mathrm{NLO}}^{\mathrm{LP}}}{\mathrm{d} \mathcal{T}}= & B_{a}^{(1)} \otimes B_{b}^{(0)} \otimes S^{(0)} \otimes H^{(0)} \\
& +B_{a}^{(0)} \otimes B_{b}^{(1)} \otimes S^{(0)} \otimes H^{(0)} \\
& +B_{a}^{(0)} \otimes B_{b}^{(0)} \otimes S^{(1)} \otimes H^{(0)} \\
& +B_{a}^{(0)} \otimes B_{b}^{(0)} \otimes S^{(0)} \otimes H^{(1)}
\end{aligned}
$$

We have introduced a superscript on each function to denote the order of $\alpha_{s}$ contributing to the convolution (for example, $S^{(0)}$ denotes the leading-order soft function, while $S^{(1)}$ denotes the $\mathcal{O}\left(\alpha_{s}\right)$ coefficient of the soft function).

We will now use the known results from the literature to separately derive the contributions above. We begin by compiling the various functions required in the factorization theorem. For simplicity, we focus in this section on those terms that involve the gluon distribution function. Contributions involving the quark distribution functions can be obtained in an identical fashion.

(i) The beam function is a nonperturbative object that can be written as a convolution of perturbative matching coefficients with the usual parton distribution functions: 


$$
B_{a}\left(t_{a}, x_{a}, \mu\right)=\sum_{i} \int_{x_{a}}^{1} \frac{\mathrm{d} z_{a}}{z_{a}} \mathcal{I}_{a i}\left(t_{a}, z_{a}, \mu\right) f_{i}\left(\frac{x_{a}}{z_{a}}, \mu\right)
$$

The matching coefficients have an expansion in $\alpha_{s}$ :

$$
\mathcal{I}_{i j}=\sum_{n=0}^{\infty}\left(\frac{\alpha_{s}}{4 \pi}\right)^{n} \mathcal{I}_{i j}^{(n)}
$$

A similar expansion for the beam function can be obtained upon substituting the matching coefficients into Eq. (10):

$$
B_{a}\left(t, x_{a}, \mu\right)=\sum_{n=0}^{\infty}\left(\frac{\alpha_{s}}{4 \pi}\right)^{n} B_{a}^{(n)}\left(t, x_{a}, \mu\right) .
$$

The matching coefficients through NNLO can be found in [34]. For our purposes we need only the LO and NLO results. Simplifying the relevant expressions from this work and keeping only those terms containing the gluon PDF for simplicity, we find

$$
\begin{aligned}
B_{g}^{(0)}\left(t_{a}, x_{a}, \mu\right)= & \delta\left(t_{a}\right) f_{g}\left(x_{a}\right), \\
B_{g}^{(1)}\left(t_{a}, x_{a}, \mu\right)= & \left(4 C_{A}\right) \int_{x_{a}}^{1} \frac{\mathrm{d} z_{a}}{z_{a}} f_{g}\left(\frac{x_{a}}{z_{a}}\right)\left\{\frac{1}{\mu^{2}} \mathcal{L}_{1}\left(\frac{t_{a}}{\mu^{2}}\right) \delta\left(1-z_{a}\right)+\frac{1}{\mu^{2}} \mathcal{L}_{0}\left(\frac{t_{a}}{\mu^{2}}\right) \mathcal{L}_{0}\left(1-z_{a}\right) \frac{\left(1-z_{a}+z_{a}^{2}\right)^{2}}{z_{a}}\right. \\
& \left.+\frac{\delta\left(t_{a}\right)}{2 Q_{a}}\left[2\left(\mathcal{L}_{1}\left(1-z_{a}\right)-\log z_{a} \mathcal{L}_{0}\left(1-z_{a}\right)\right) \frac{\left(1-z_{a}+z_{a}^{2}\right)^{2}}{z_{a}}-\frac{\pi^{2}}{6} \delta\left(1-z_{a}\right)\right]\right\},
\end{aligned}
$$

where the plus distribution is written as

$$
\mathcal{L}_{n}(x)=\left[\frac{\theta(x) \log ^{n} x}{x}\right]_{+} .
$$

(ii) The soft function for 0 -jettiness at the NLO level can be obtained from Ref. [35]. The tree-level result is

$$
S^{(0)}=\delta\left(\mathcal{T}_{a}-\frac{t_{a}}{Q_{a}}\right) \delta\left(\mathcal{T}_{b}-\frac{t_{b}}{Q_{b}}\right),
$$

while the one-loop correction can be parametrized as

$$
S^{(1)}=\sum_{i \neq j} \mathbf{T}_{i} \cdot \mathbf{T}_{j} S_{i j}^{(1)} .
$$

In our case, the indices $i$ and $j$ can only take the values 1,2 . We have

$$
\sum_{i \neq j} \mathbf{T}_{i} \cdot \mathbf{T}_{j}=-C_{A} .
$$

Furthermore, we only have the hemisphere contribution to the soft function in the language of Ref. [35]:

$$
\begin{aligned}
S_{a b}^{(1)}= & \frac{\alpha_{s}(\mu)}{4 \pi}\left[\frac{8}{\sqrt{\hat{s}_{a b}} \mu} \mathcal{L}_{1}\left(\frac{\mathcal{T}_{a}-\frac{t_{a}}{Q_{a}}}{\sqrt{\hat{s}_{a b}} \mu}\right)\right. \\
& \left.-\frac{\pi^{2}}{6} \delta\left(\mathcal{T}_{a}-\frac{t_{a}}{Q_{a}}\right)\right] \delta\left(\mathcal{T}_{b}-\frac{t_{b}}{Q_{b}}\right) .
\end{aligned}
$$

The constant $\hat{s}_{a b}$ is

$$
\hat{s}_{a b}=2 \hat{q}_{a} \cdot \hat{q}_{b}=\frac{s x_{a} x_{b}}{Q_{a} Q_{b}}=1,
$$

where the $\hat{q}_{i}$ are defined as

$$
\hat{q}_{i}^{\mu}=\frac{q_{i}^{\mu}}{Q_{i}} .
$$

We have assumed for simplicity that the normalization constants are chosen so that $Q_{a} Q_{b}=s x_{a} x_{b}$, as is the case for the leptonic and hadronic definitions of $\mathcal{T}$. There is an analogous contribution $S_{b a}^{(1)}$ where $a \leftrightarrow b$.

(iii) After renormalization the NLO hard function contains the finite contributions to the virtual corrections. We can obtain the leading-order hard function for Higgs production from [36,37]. The NLO result can be taken from Ref. [38]. The tree-level hard function is simply the tree level amplitude squared, which in the effective theory with $m_{H} \ll m_{\text {top }}$ takes the form

$$
H^{(0)}\left(p_{H}, \mu\right)=\left|\mathcal{M}_{\mathrm{Born}}\right|^{2}=\frac{\alpha_{s}^{2} m_{H}^{2} \hat{s}}{576 v^{2} \pi^{2}},
$$

where $\hat{s} \equiv x_{a} x_{b} s$. At tree level, the $d=4$ dimensional version of the Born matrix element is sufficient. At NLO, we have, in the $\overline{\mathrm{MS}}$ scheme, 


$$
\begin{aligned}
H^{(1)}\left(p_{H}, \mu\right)= & H^{(0)}\left(p_{H}, \mu\right)\left(\frac{\alpha_{s} C_{A}}{\pi}\right) \\
& \times\left\{\frac{7}{12} \pi^{2}-\frac{1}{2} \log ^{2}\left(\frac{m_{H}^{2}}{\mu^{2}}\right)\right\} .
\end{aligned}
$$

There is as additional correction in the effectivetheory Lagrangian coming from integrating out the top quark. As this term is treated identically in SCET and in the direct QCD derivation of the differential cross section we do not discuss it explicitly here. It is included in all numerical results.

We must now use these expressions in the expanded factorization formula of Eq. (9). At the NLO level there is no nontrivial convolution to perform, since in each case all but one function takes on its simple tree-level form, and all integrals can be done straightforwardly. We separately present the individual contributions to the differential cross section in $\mathcal{T}$ for the gluon-gluon partonic channel below:

$$
\begin{aligned}
& B_{g}^{(1)} \otimes B_{g}^{(0)} \otimes S^{(0)} \otimes H^{(0)}=\left(\frac{\alpha_{s} C_{A}}{\pi}\right) \int \mathrm{d} x_{a} \int \mathrm{d} x_{b} \int \mathrm{d} \Phi_{H}\left|\mathcal{M}_{\mathrm{Born}}\right|^{2} f_{g}\left(x_{b}\right) \int_{x_{a}} \frac{\mathrm{d} z_{a}}{z_{a}} f_{g}\left(\frac{x_{a}}{z_{a}}\right) \\
& \times\left\{\frac{Q_{a}}{\mu^{2}} \mathcal{L}_{1}\left(\frac{Q_{a} \mathcal{T}}{\mu^{2}}\right) \delta\left(1-z_{a}\right)+\frac{Q_{a}}{\mu^{2}} \mathcal{L}_{0}\left(\frac{Q_{a} \mathcal{T}}{\mu^{2}}\right) \mathcal{L}_{0}\left(1-z_{a}\right) \frac{\left(1-z_{a}+z_{a}^{2}\right)^{2}}{z_{a}}\right. \\
&\left.+\delta(\mathcal{T})\left[\left(\mathcal{L}_{1}\left(1-z_{a}\right)-\log z_{a} \mathcal{L}_{0}\left(1-z_{a}\right)\right) \frac{\left(1-z_{a}+z_{a}^{2}\right)^{2}}{z_{a}}-\frac{\pi^{2}}{12} \delta\left(1-z_{a}\right)\right]\right\} ; \\
& B_{g}^{(0)} \otimes B_{g}^{(0)} \otimes S^{(1)} \otimes H^{(0)}=\left(\frac{\alpha_{s} C_{A}}{\pi}\right) \int \mathrm{d} x_{a} \int \mathrm{d} x_{b} \int \mathrm{d} \Phi_{H}\left|\mathcal{M}_{\mathrm{Born}}\right|^{2} f_{g}\left(x_{a}\right) f_{g}\left(x_{b}\right)\left\{-\frac{4}{\mu} \mathcal{L}_{1}\left(\frac{\mathcal{T}}{\mu}\right)+\frac{\pi^{2}}{12} \delta(\mathcal{T})\right\} ; \\
& B_{g}^{(0)} \otimes B_{g}^{(0)} \otimes S^{(0)} \otimes H^{(1)}=\left(\frac{\alpha_{s} C_{A}}{\pi}\right) \int \mathrm{d} x_{a} \int \mathrm{d} x_{b} \int \mathrm{d} \Phi_{H}\left|\mathcal{M}_{\mathrm{Born}}\right|^{2} f_{g}\left(x_{a}\right) f_{g}\left(x_{b}\right) \delta(\mathcal{T})\left[\frac{7}{12} \pi^{2}-\frac{1}{2} \log ^{2}\left(\frac{m_{H}^{2}}{\mu^{2}}\right)\right]
\end{aligned}
$$

There is a fourth contribution $B_{g}^{(0)} \otimes B_{g}^{(1)} \otimes S^{(0)} \otimes H^{(0)}$ which can be obtained from Eq. (23) with the substitution $a \leftrightarrow b$.

\section{DIRECT QCD DERIVATION OF LEADING AND SUBLEADING POWER}

In order to study in detail the structure of the power corrections to the 0 -jettiness factorization formula it is useful to expand the cross section directly in QCD. It turns out to be possible to obtain the full $\mathcal{O}\left(\mathcal{T}^{\text {cut }}\right)$ power corrections, not just the logarithmically enhanced terms studied previously. We will derive here the leading-power result as well to compare to the SCET expression of the previous section. This serves as a check on our result, and we hope that it is also useful to the reader interested in understanding the connection between the SCET formalism and standard QCD. For clarity, the $\mathcal{T}$ behavior of the coefficients at leading-power and next-to-leading-power is shown in Table I. We show the power counting both for the differential cross section in $\mathcal{T}$ and for the result integrated up to a cutoff $\mathcal{T}^{\text {cut }}$. We begin by discussing the factorization of the phase space and the expansion of the matrix elements in $\mathcal{T}$. Most aspects of our derivation are applicable to arbitrary 0 -jettiness processes and not just gluon-fusion Higgs production. We identify in the text which parts of our derivation are generic and which parts must be modified for other processes.

\section{A. Factorization of the phase space}

The SCET result for the below-cut region in Eq. (7) is written in a form that explicitly factors out the Bornlevel phase space. This is possible directly in QCD with straightforward changes of variables. The strategy used here is to absorb the kinematics of the emitted final-state gluon into one of the two incoming gluons. We begin by deriving the Born-level phase space for the leading-order process $g\left(q_{a}\right)+g\left(q_{b}\right) \rightarrow H$, including also the convolution over parton distribution functions and the flux factor. The kinematics for the initial state was described in Sec. II, from which we find

TABLE I. Power counting in $\mathcal{T}$ of the NLO cross section differential in $\mathcal{T}$ (left) and integrated up to $\mathcal{T}^{\text {cut }}$ (right), at leadingpower, next-to-leading-power and next-to-next-to-leading power. We note that there is no NNLL contribution at NLO in $\alpha_{s}$.

\begin{tabular}{lccc}
\hline \hline$\frac{\mathrm{d} \sigma_{\mathrm{NLO}}}{\mathrm{d} \mathcal{T}}$ & $\mathrm{LL}$ & $\mathrm{NLL}$ & $\mathrm{NNLL}$ \\
\hline $\mathrm{LP}$ & {$\left[\frac{\log \mathcal{T}}{\mathcal{T}}\right]_{+}$} & {$\left[\frac{1}{\mathcal{T}}\right]_{+}$} & $\delta(\mathcal{T})$ \\
$\mathrm{NLP}$ & $\log \mathcal{T}$ & 1 & \\
\hline \hline$\sigma_{\mathrm{NLO}}$ & $\mathrm{LL}$ & $\mathrm{NLL}$ & $\mathrm{NNLL}$ \\
\hline $\mathrm{LP}$ & $\log ^{2} \mathcal{T}^{\text {cut }}$ & $\log \mathcal{T}^{\text {cut }}$ & 1 \\
$\mathrm{NLP}$ & $\mathcal{T}^{\text {cut }} \log \mathcal{T}^{\text {cut }}$ & $\mathcal{T}^{\text {cut }}$ & \\
\hline \hline
\end{tabular}




$$
\begin{aligned}
\mathrm{PS}_{\mathrm{Born}} & =(2 \pi) \int_{0}^{1} \mathrm{~d} x_{a} \int_{0}^{1} \mathrm{~d} x_{b} \frac{f_{g}\left(x_{a}\right) f_{g}\left(x_{b}\right)}{2 s x_{a} x_{b}} \int \mathrm{d}^{d} p_{H} \delta\left(p_{H}^{2}-m_{H}^{2}\right) \delta^{(d)}\left(q_{a}+q_{b}-p_{H}\right) \\
& =(2 \pi) \int_{0}^{1} \mathrm{~d} x_{a} \int_{0}^{1} \mathrm{~d} x_{b} \frac{f_{g}\left(x_{a}\right) f_{g}\left(x_{b}\right)}{2 s x_{a} x_{b}} \delta\left(s x_{a} x_{b}-m_{H}^{2}\right) .
\end{aligned}
$$

At NLO we consider the partonic process $g\left(q_{a}^{\prime}\right)+g\left(q_{b}^{\prime}\right) \rightarrow H+g\left(p_{3}\right)$. The same approach is applicable to the other NLO partonic channels, which are $q\left(q_{a}^{\prime}\right)+g\left(q_{b}^{\prime}\right) \rightarrow H+q\left(p_{3}\right), g\left(q_{a}^{\prime}\right)+q\left(q_{b}^{\prime}\right) \rightarrow H+g\left(p_{3}\right)$, and $q\left(q_{a}^{\prime}\right)+\bar{q}\left(q_{b}^{\prime}\right) \rightarrow$ $H+g\left(p_{3}\right)$. For simplicity we only present the derivation of the most complicated $g g$ case here. The subleading power results for the other partonic channels are given in the Appendix. We have relabeled the initial-state gluon momenta to distinguish them from the momenta entering the 0 -jettiness definition of Eq. (2). Denoting the Bjorken- $x$ values as $\xi_{a}$ and $\xi_{b}$ to distinguish them from those entering the 0-jettiness definition in Eq. (2), we have

$$
\begin{aligned}
\mathrm{PS}_{\mathrm{NLO}}= & \frac{\mu_{0}^{2 \varepsilon}}{(2 \pi)^{d-2}} \int_{0}^{1} \mathrm{~d} \xi_{a} \int_{0}^{1} \mathrm{~d} \xi_{b} \frac{f_{g}\left(\xi_{a}\right) f_{g}\left(\xi_{b}\right)}{2 s \xi_{a} \xi_{b}} \int \mathrm{d}^{d} p_{3} \delta\left(p_{3}^{2}\right) \\
& \times \int \mathrm{d}^{d} p_{H} \delta\left(p_{H}^{2}-m_{H}^{2}\right) \delta^{(d)}\left(q_{a}^{\prime}+q_{b}^{\prime}-p_{3}-p_{H}\right) \Theta\left(s \xi_{a} \xi_{b}-m_{H}^{2}\right) \Theta\left(E_{H}\right) \\
= & \frac{\mu_{0}^{2 \varepsilon}}{(2 \pi)^{d-2}} \int_{0}^{1} \mathrm{~d} \xi_{a} \int_{0}^{1} \mathrm{~d} \xi_{b} \frac{f_{g}\left(\xi_{a}\right) f_{g}\left(\xi_{b}\right)}{2 s \xi_{a} \xi_{b}} \int \mathrm{d}^{d} p_{3} \delta\left(p_{3}^{2}\right) \delta\left(\hat{s}-m_{H}^{2}-2 q_{a}^{\prime} \cdot p_{3}-2 q_{b}^{\prime} \cdot p_{3}\right) .
\end{aligned}
$$

We have included $\mu_{0}$, the usual dimensional-regularization mass scale, in the phase space. In the second line we have used momentum conservation to remove the $p_{H}$ integral, and have suppressed the energy theta-function for notational simplicity. We use a Sudakov decomposition for the gluon momentum $p_{3}$ :

$$
\begin{aligned}
p_{3}^{\mu} & =\frac{\bar{n} \cdot p_{3}}{2} n^{\mu}+\frac{n \cdot p_{3}}{2} \bar{n}^{\mu}+p_{3 T}^{\mu} \\
\int \mathrm{d}^{d} p_{3} \delta\left(p_{3}^{2}\right) & =\frac{\Omega_{d-2}}{4} \int \mathrm{d}\left(\bar{n} \cdot p_{3}\right)\left(\bar{n} \cdot p_{3}\right)^{-\varepsilon} \int \mathrm{d}\left(n \cdot p_{3}\right)\left(n \cdot p_{3}\right)^{-\varepsilon} .
\end{aligned}
$$

We now incorporate the definition of 0 -jettiness into the phase space. The emitted gluon $p_{3}$ can be closer to either the $n$ or $\bar{n}$ direction, leading to different expressions for $\mathcal{T}$ according to the definition in Eq. (2). We will assume the first case. The second case can be easily obtained by exchanging $a \leftrightarrow b$. In this region we have

$$
\mathcal{T}=\frac{x_{a} \sqrt{s}}{Q_{a}} n \cdot p_{3}
$$

We note that $\mathcal{T}$ has been defined using the $q_{a}$ and $q_{b}$ defined in Eq. (3), which depend upon the Born-level Bjorken- $x$ values $x_{a}$ and $x_{b}$. We will see how to define these quantities at NLO shortly. We can now write down the differential phase space for this partition as

$$
\begin{aligned}
\frac{\mathrm{dPS}_{\mathrm{NLO}}^{(a)}}{\mathrm{d} \mathcal{T}}= & \frac{\mathcal{T}^{-\varepsilon}}{8 \pi} \frac{\left(4 \pi \mu_{0}^{2}\right)^{\varepsilon}}{\Gamma(1-\varepsilon)} \int_{0}^{1} \mathrm{~d} \xi_{a} \int_{0}^{1} \mathrm{~d} \xi_{b} \frac{f_{g}\left(\xi_{a}\right) f_{g}\left(\xi_{b}\right)}{2 s \xi_{a} \xi_{b}}\left(\frac{Q_{a}}{x_{a} \sqrt{s}}\right)^{1-\varepsilon} \\
& \times \int \mathrm{d}\left(\bar{n} \cdot p_{3}\right)\left(\bar{n} \cdot p_{3}\right)^{-\varepsilon} \delta\left(s \xi_{a} \xi_{b}-m_{H}^{2}-Q_{a} \frac{\xi_{a}}{x_{a}} \mathcal{T}-\left(\bar{n} \cdot p_{3}\right) \xi_{b} \sqrt{s}\right) .
\end{aligned}
$$

We rescale $\bar{n} \cdot p_{3}$ so that it ranges from 0 to 1 using the variable change

$$
\bar{n} \cdot p_{3}=\sqrt{s} \xi_{a}\left(1-z_{a}\right)
$$

leading to the expression 


$$
\begin{aligned}
\frac{\mathrm{dPS}_{\mathrm{NLO}}^{(a)}}{\mathrm{d} \mathcal{T}}= & \frac{\mathcal{T}^{-\varepsilon}}{8 \pi} \frac{\left(4 \pi \mu_{0}^{2}\right)^{\varepsilon}}{\Gamma(1-\varepsilon)} \int_{0}^{1} \mathrm{~d} \xi_{a} \\
& \times \int_{0}^{1} \mathrm{~d} \xi_{b} \frac{f_{g}\left(\xi_{a}\right) f_{g}\left(\xi_{b}\right)}{2 s \xi_{a} \xi_{b}}\left(\frac{Q_{a} \xi_{a}}{x_{a}}\right)^{1-\varepsilon} \\
& \times \int \mathrm{d} z_{a}\left(1-z_{a}\right)^{-\varepsilon} \delta\left(s \xi_{a} \xi_{b} z_{a}-m_{H}^{2}-Q_{a} \frac{\xi_{a}}{x_{a}} \mathcal{T}\right) .
\end{aligned}
$$

At this point we wish to identify and factor out the Born phase space defined in Eq. (26) from this expression. We can do so with the following variable changes, which also serve the purpose of defining the $x_{a}$ and $x_{b}$ that appear in the 0 -jettiness definition:

$$
\xi_{a}=\frac{s x_{a}^{2} x_{b}}{s x_{a} x_{b} z_{a}-Q_{a} \mathcal{T}} \quad \xi_{b}=x_{b}
$$

These transformations force the delta function in Eq. (32) into the form which appears in the LO phase space. We note one critical point that appears when we perform this variable change. In general, $Q_{a}$ is a function of $x_{a}$ (for example, for the hadronic $\mathcal{T}$ it is $\left.Q_{a}\left(x_{a}\right)=\sqrt{s} x_{a}\right)$. Similarly, $Q_{b}=Q_{a}\left(x_{b}\right)$ in general. Therefore derivatives of the $Q_{i}$ appear when changing variables from $\xi_{a}$ to $x_{a}$. As we discuss later in more detail, the contribution of this derivative to the power corrections for hadronic $\mathcal{T}$ appears to have been neglected in the derivation of Refs. [30,32]. Upon making these variable changes and expanding the phase-space measure to $\mathcal{O}(\mathcal{T})$, we have

$$
\begin{aligned}
\frac{\mathrm{dPS}_{\mathrm{NLO}}^{(a)}}{\mathrm{d} \mathcal{T}}= & \frac{\mathcal{T}^{-\varepsilon}}{8 \pi} \frac{\left(4 \pi \mu_{0}^{2}\right)^{\varepsilon}}{\Gamma(1-\varepsilon)} \int_{0}^{1} \mathrm{~d} x_{a} \int_{0}^{1} \mathrm{~d} x_{b} \frac{f_{g}\left(x_{b}\right)}{2 s x_{a} x_{b}} \delta\left(s x_{a} x_{b}-m_{H}^{2}\right) \int_{x_{a}+\frac{Q_{H} \mathcal{T}}{m_{H}^{2}}}^{1-\frac{\tau Q_{b}}{z_{a}^{2}}} \frac{\mathrm{d} z_{a}}{z_{a}}\left(Q_{a}\right)^{1-\varepsilon}\left(\frac{1-z_{a}}{z_{a}}\right)^{-\varepsilon} \\
& \times\left\{f_{g}\left(\frac{x_{a}}{z_{a}}\right)+\frac{\mathcal{T}}{m_{H}^{2} z_{a}^{2}}\left[\left(Q_{a}^{\prime} z_{a} x_{a}-Q_{a} z_{a} \varepsilon\right) f_{g}\left(\frac{x_{a}}{z_{a}}\right)+Q_{a} x_{a} f_{g}^{\prime}\left(\frac{x_{a}}{z_{a}}\right)\right]+\mathcal{O}\left(\mathcal{T}^{2}\right)\right\} .
\end{aligned}
$$

The prime denotes a derivative with respect to $x_{a}$. This has the desired factorized Born-level phase space. We note that the lower limit on the $z_{a}$ integral comes from the requirement $\xi_{a} \leq 1$, while the upper limit comes from the 0 -jettiness requirement

$$
\mathcal{T} \leq \frac{x_{b} \sqrt{s} \bar{n} \cdot p_{3}}{Q_{b}} \Rightarrow z_{a} \leq 1-\frac{\mathcal{T} Q_{b}}{m_{H}^{2}}+\mathcal{O}\left(\mathcal{T}^{2}\right) .
$$

We have checked that the $z_{a}$ integral can be extended down to $x_{a}$, since the region between $x_{a}$ and $x_{a}+\frac{Q_{a} \mathcal{T}}{m_{H}^{2}}$ does not contribute to the LL nor to the NLL power corrections. We will express our results in the usual $\overline{\mathrm{MS}}$ scheme by replacing

$$
\left(4 \pi \mu_{0}^{2}\right)^{\varepsilon}=\left(e^{\gamma_{E}} \mu^{2}\right)^{\varepsilon} .
$$

We note that this factorization of the phase space is valid for any process using 0 -jettiness as a resolution parameter.

\section{B. Expansion of the matrix elements}

We now consider the matrix elements for the NLO real-emission correction $g g \rightarrow H g$, expanded in $\mathcal{T}$. This partonic channel is numerically the most important contribution to gluon-fusion Higgs production. Results for the other channels are given in the Appendix. We note that the structure of the power corrections takes the form of leadingpower phase space combined with subleading power matrix elements, plus subleading power phase space combined with leading-power matrix elements. While the exact expressions for the matrix elements are of course process-dependent, the structure of the power corrections is the same for any 0 -jettiness process.

The Born matrix element for the partonic process $g g \rightarrow$ $H$ in $d$ dimensions is $[36,37]$

$$
|\mathcal{M}(g g \rightarrow H)|^{2}=\frac{\alpha_{s}^{2} m_{H}^{4}}{576 \pi^{2} v^{2}}\left(\frac{\mu^{2}}{m_{t}^{2}}\right)^{2 \varepsilon} \frac{\Gamma^{2}(1+\varepsilon)}{1-\varepsilon} .
$$

For the real emission of a gluon, we have

$$
|\mathcal{M}(g g \rightarrow H g)|^{2}=\frac{8 C_{A} \pi \alpha_{s}}{m_{H}^{4}(1-\varepsilon)}|\mathcal{M}(g g \rightarrow H)|^{2}\left[\frac{m_{H}^{8}+s_{12}^{4}+s_{13}^{4}+s_{23}^{4}}{s_{12} s_{13} s_{23}}(1-2 \varepsilon)+\frac{\varepsilon}{2} \frac{\left(m_{H}^{4}+s_{12}^{2}+s_{13}^{2}+s_{23}^{2}\right)^{2}}{s_{12} s_{13} s_{23}}\right] .
$$

The invariants that appear in the matrix elements are given in our phase-space parametrization by

$$
s_{12}=s \xi_{a} \xi_{b}=\frac{m_{H}^{2}}{z_{a}-\frac{Q a \mathcal{T}}{m_{H}^{2}}}, \quad s_{13}=-\frac{Q_{a} \mathcal{T}}{z_{a}-\frac{Q a \mathcal{T}}{m_{H}^{2}}}, \quad s_{23}=-m_{H}^{2} \frac{1-z_{a}}{z_{a}-\frac{Q a \mathcal{T}}{m_{H}^{2}}}
$$


Therefore, we can write the NLO matrix element including both the leading power in $\mathcal{T}$ and the first correction as

$$
|\mathcal{M}(g g \rightarrow H g)|^{2}=|\mathcal{M}(g g \rightarrow H)|^{2}\left(16 C_{A} \alpha_{s} \pi\right)\left\{\frac{1}{Q_{a} \mathcal{T}} \frac{\left(1-z_{a}+z_{a}^{2}\right)^{2}}{\left(1-z_{a}\right) z_{a}}+\frac{1}{m_{H}^{2}}\left[5-\frac{1}{1-z_{a}}+\frac{1}{z_{a}^{2}}-\frac{1}{z_{a}}+z_{a}-\frac{2}{1-\varepsilon}\right]+\mathcal{O}(\mathcal{T})\right\}
$$

\section{Derivation of the leading-power result}

We begin by deriving the leading-power expression to compare with our result of Sec. III. To obtain this term we take the leading-power expression for both the phase space and the matrix element. We can write the differential cross section for the first phase-space partition as

$$
\begin{aligned}
\frac{\mathrm{d} \sigma_{\mathrm{NLO}}^{\mathrm{LP},(a)}}{\mathrm{d} \mathcal{T}}= & \left(\frac{C_{A} \alpha_{s}}{\pi}\right) \frac{\left(e^{\gamma_{E}}\right)^{\varepsilon}}{\Gamma(1-\varepsilon)} \int_{0}^{1} \mathrm{~d} x_{a} \int_{0}^{1} \mathrm{~d} x_{b} \frac{(2 \pi) f_{g}\left(x_{b}\right)}{2 s x_{a} x_{b}}|\mathcal{M}(g g \rightarrow H)|^{2} \delta\left(s x_{a} x_{b}-m_{H}^{2}\right) \\
& \times\left(\frac{\mathcal{T} Q_{a}}{\mu^{2}}\right)^{-1-\varepsilon} \frac{Q_{a}}{\mu^{2}} \int_{x_{a}}^{1-\frac{\tau Q_{b}}{m_{H}^{2}}} \frac{\mathrm{d} z_{a}}{z_{a}} f_{g}\left(\frac{x_{a}}{z_{a}}\right)\left(1-z_{a}\right)^{-1-\varepsilon} z_{a}^{\varepsilon} \frac{\left(1-z_{a}+z_{a}^{2}\right)^{2}}{z_{a}} .
\end{aligned}
$$

To proceed we divide the integral in $z_{a}$ into two regions as follows:

$$
\int_{x_{a}}^{1-\frac{\tau Q_{b}}{m_{H}^{2}}} \mathrm{~d} z_{a}=\int_{x_{a}}^{1} \mathrm{~d} z_{a}-\int_{1-\frac{\tau Q_{b}}{m_{H}^{2}}}^{1} \mathrm{~d} z_{a}
$$

We can now establish the connection between the direct QCD calculation performed here and the SCET result of Sec. III. The first integral will give us the beam function contribution of SCET, while the second integral will give us the soft function contribution. We expand in $\varepsilon$ using

$$
\begin{gathered}
\frac{Q_{a}}{\mu^{2}}\left(\frac{Q_{a} \mathcal{T}}{\mu^{2}}\right)^{-1-\varepsilon} \rightarrow-\frac{\delta(\mathcal{T})}{\varepsilon}+\frac{Q_{a}}{\mu^{2}} \mathcal{L}_{0}\left(\frac{Q_{a} \mathcal{T}}{\mu^{2}}\right)-\varepsilon \frac{Q_{a}}{\mu^{2}} \mathcal{L}_{1}\left(\frac{Q_{a} \mathcal{T}}{\mu^{2}}\right), \\
\left(1-z_{a}\right)^{-1-\varepsilon} z_{a}^{\varepsilon} \rightarrow-\frac{\delta\left(1-z_{a}\right)}{\varepsilon}+\mathcal{L}_{0}\left(1-z_{a}\right)-\varepsilon\left[\mathcal{L}_{1}\left(1-z_{a}\right)-\log z_{a} \mathcal{L}_{0}\left(1-z_{a}\right)\right],
\end{gathered}
$$

Using these expressions the differential cross section can be written as

$$
\begin{aligned}
\frac{\mathrm{d} \sigma_{\text {beam }}^{\mathrm{LP},(a)}}{\mathrm{d} \mathcal{T}}= & \left(\frac{\alpha_{s} C_{A}}{\pi}\right) \int_{0}^{1} \mathrm{~d} x_{a} \int_{0}^{1} \mathrm{~d} x_{b}(2 \pi) \frac{f_{g}\left(x_{b}\right)}{2 s x_{a} x_{b}}\left|\mathcal{M}_{\text {Born }}\right|^{2} \delta\left(s x_{a} x_{b}-m_{H}^{2}\right) \int_{x_{a}}{ }^{1} \frac{\mathrm{d} z_{a}}{z_{a}} f_{g}\left(\frac{x_{a}}{z_{a}}\right) \\
& \times\left\{\frac{1}{\varepsilon^{2}} \delta(\mathcal{T}) \delta\left(1-z_{a}\right)-\frac{1}{\varepsilon} \mathcal{L}_{0}\left(1-z_{a}\right) \frac{\left(1-z_{a}+z_{a}^{2}\right)^{2}}{z_{a}} \delta(\mathcal{T})-\frac{1}{\varepsilon}\left(\frac{Q_{a}}{\mu^{2}}\right) \mathcal{L}_{0}\left(\frac{Q_{a} \mathcal{T}}{\mu^{2}}\right) \delta\left(1-z_{a}\right)\right. \\
& +\left(\frac{Q_{a}}{\mu^{2}}\right) \mathcal{L}_{1}\left(\frac{Q_{a} \mathcal{T}}{\mu^{2}}\right) \delta\left(1-z_{a}\right)+\left(\frac{Q_{a}}{\mu^{2}}\right) \mathcal{L}_{0}\left(\frac{Q_{a} \mathcal{T}}{\mu^{2}}\right) \mathcal{L}_{0}\left(1-z_{a}\right) \frac{\left(1-z_{a}+z_{a}^{2}\right)^{2}}{z_{a}} \\
& \left.-\frac{\pi^{2}}{12} \delta(\mathcal{T}) \delta\left(1-z_{a}\right)+\left[\mathcal{L}_{1}\left(1-z_{a}\right)-\log z_{a} \mathcal{L}_{0}\left(1-z_{a}\right)\right] \frac{\left(1-z_{a}+z_{a}^{2}\right)^{2}}{z_{a}} \delta(\mathcal{T})\right\}
\end{aligned}
$$

The term inside the braces, together with the overall factor outside of the integral, is exactly the bare gluon beam function [33]. The finite terms are the same that we found in Eq. (23) by expanding the SCET factorization formula. In the effective theory the poles would be removed by a separate renormalization of the beam function and by matching to the PDFs. In our QCD calculation they cancel upon adding all contributions to the cross section, including the mass factorization counterterms.

We now consider the second region of the $z_{a}$ integral in Eq. (42). We can expand the integrand around $z_{a}=1$ :

$$
\int_{1-\frac{\tau Q_{b}}{m_{H}^{2}}}^{1} \mathrm{~d} z_{a}\left\{\left(1-z_{a}\right)^{-1-\varepsilon} f\left(x_{a}\right)+\left(1-z_{a}\right)^{-\varepsilon}\left[\varepsilon f\left(x_{a}\right)-x_{a} f_{g}^{\prime}\left(x_{a}\right)\right]\right\}
$$

The first term in this expansion contributes to the leading-power result. The second term contributes only to subleading power, and will be needed in the next subsection. At leading power, the following integral is needed: 


$$
\int_{1-\frac{\mathcal{T} Q_{b}}{m_{H}^{2}}}^{1} \mathrm{~d} z_{a}\left(1-z_{a}\right)^{-1-\varepsilon}=-\frac{1}{\varepsilon}\left(\frac{\mathcal{T} Q_{b}}{m_{H}^{2}}\right)^{-\varepsilon} .
$$

We compile here for use in the subleading power derivation the following result as well:

$$
\int_{1-\frac{\tau Q_{b}}{m_{H}^{2}}}^{1} \mathrm{~d} z_{a}\left(1-z_{a}\right)^{-\varepsilon}=\frac{\left(\frac{Q_{b} \mathcal{T}}{m_{H}^{2}}\right)^{1-\varepsilon}}{1-\varepsilon} .
$$

We again assume for simplicity that $Q_{a} Q_{b}=m_{H}^{2}$ as we did in Sec. III, leading to the result:

$$
\begin{aligned}
\frac{\mathrm{d} \sigma_{\text {soft }}^{\mathrm{LP},(a)}}{\mathrm{d} \mathcal{T}}= & \left(\frac{\alpha_{s} C_{A}}{\pi}\right) \int_{0}^{1} \mathrm{~d} x_{a} \\
& \times \int_{0}^{1} \mathrm{~d} x_{b}(2 \pi) \frac{f_{g}\left(x_{a}\right) f_{g}\left(x_{b}\right)}{2 s x_{a} x_{b}}\left|\mathcal{M}_{\mathrm{Born}}\right|^{2} \\
& \times\left\{-\frac{1}{2 \varepsilon^{2}} \delta(\mathcal{T})+\frac{1}{\varepsilon \mu} \mathcal{L}_{0}\left(\frac{\mathcal{T}}{\mu}\right)\right. \\
& \left.-\frac{2}{\mu} \mathcal{L}_{1}\left(\frac{\mathcal{T}}{\mu}\right)+\frac{\pi^{2}}{24} \delta(\mathcal{T})\right\}
\end{aligned}
$$

We can again compare the finite part of this result to the SCET soft-function contribution of Eq. (24). This is equal to half of the result there. The remaining factor of two is provided by the other phase-space partition, establishing the exact correspondence between the QCD calculation and the SCET derivation. We have performed this check for the other partonic channels as well.

It only remains to consider the virtual corrections to the cross section and to establish the cancellation of poles. Since the hard function of SCET is exactly the finite part of the virtual corrections in QCD as can be checked by comparing Refs. [38,39], the correspondence between the QCD virtual corrections and the SCET hard-function contribution of Eq. (25) is obvious.

We now focus on the poles, since we have already established the agreement of the finite parts between SCET and QCD for the separate contributions. We can combine both beam regions and both soft contributions to get the full result for the real-emission correction at leading power:

$$
\frac{\mathrm{d} \sigma_{\text {real }}^{\mathrm{LP}}}{\mathrm{d} \mathcal{T}}=\frac{\mathrm{d} \sigma_{\text {beam }}^{\mathrm{LP},(a)}}{\mathrm{d} \mathcal{T}}+\frac{\mathrm{d} \sigma_{\text {soft }}^{\mathrm{LP},(a)}}{\mathrm{d} \mathcal{T}}+\frac{\mathrm{d} \sigma_{\text {beam }}^{\mathrm{LP}(b)}}{\mathrm{d} \mathcal{T}}+\frac{\mathrm{d} \sigma_{\text {soft }}^{\mathrm{LP},(b)}}{\mathrm{d} \mathcal{T}}
$$

Combining these terms we arrive at

$$
\begin{aligned}
\frac{\mathrm{d} \sigma_{\text {real }}^{\mathrm{LP}}}{\mathrm{d} \mathcal{T}}= & \left(\frac{\alpha_{s} C_{A}}{\pi}\right) \int_{0}^{1} \mathrm{~d} x_{a} \int_{0}^{1} \mathrm{~d} x_{b} \frac{(2 \pi)\left|\mathcal{M}_{\mathrm{Born}}\right|^{2}}{2 s x_{a} x_{b}} \delta\left(s x_{a} x_{b}-m_{H}^{2}\right) \int_{x_{a}}^{1} \frac{\mathrm{d} z_{a}}{z_{a}} f_{g}\left(\frac{x_{a}}{z_{a}}\right) \int_{x_{b}}^{1} \frac{\mathrm{d} z_{b}}{z_{b}} f_{g}\left(\frac{x_{a}}{z_{b}}\right) \\
& \times \delta(\mathcal{T})\left\{\delta\left(1-z_{a}\right) \delta\left(1-z_{b}\right)\left[\frac{1}{\varepsilon^{2}}-\frac{1}{\varepsilon} \log \left(\frac{m_{H}^{2}}{\mu^{2}}\right)\right]-\frac{1}{\varepsilon} \mathcal{L}_{0}\left(1-z_{a}\right) \frac{\left(1-z_{a}+z_{a}^{2}\right)^{2}}{z_{a}}-\frac{1}{\varepsilon} \mathcal{L}_{0}\left(1-z_{b}\right) \frac{\left(1-z_{b}+z_{b}^{2}\right)^{2}}{z_{b}}\right\} .
\end{aligned}
$$

We note that in writing this expression we have made use of the following relation:

$$
2 \mathcal{L}_{0}\left(\frac{\mathcal{T}}{\mu}\right)-\left(\frac{Q_{a}}{\mu^{2}}\right) \mathcal{L}_{0}\left(\frac{Q_{a} \mathcal{T}}{\mu^{2}}\right)-\left(\frac{Q_{b}}{\mu^{2}}\right) \mathcal{L}_{0}\left(\frac{Q_{b} \mathcal{T}}{\mu^{2}}\right)=-\frac{1}{\varepsilon} \log \left(\frac{Q_{a} Q_{b}}{\mu^{2}}\right) \delta(\mathcal{T})=-\frac{1}{\varepsilon} \log \left(\frac{m_{H}^{2}}{\mu^{2}}\right) \delta(\mathcal{T})
$$

This is the expression for the poles of the real-emission corrections. The first two terms cancel against the virtual corrections, whose pole structure at NLO in the $\overline{\mathrm{MS}}$ scheme is given by [39]

$$
\sigma_{V}=\sigma_{\mathrm{Born}}\left(\frac{\alpha_{s} C_{A}}{\pi}\right)\left(\frac{\mu^{2}}{m_{H}^{2}}\right)^{\varepsilon}\left[-\frac{1}{\varepsilon^{2}}\right]=\sigma_{\mathrm{Born}}\left(\frac{\alpha_{s} C_{A}}{\pi}\right)\left[-\frac{1}{\varepsilon^{2}}+\frac{1}{\varepsilon} \log \left(\frac{m_{H}^{2}}{\mu^{2}}\right)\right] .
$$

The second two terms are removed by mass factorization into the initial-state PDFs, leaving a finite cross section. This completely establishes both the cancellation of poles and the equivalence of QCD and SCET at leading power in $\mathcal{T}$. We note that we have not considered the additional contribution in the Higgs effective theory coming from integrating out the top quark, since it is treated identically in both a direct QCD calculation and in the effective theory.

\section{Derivation of the subleading-power result}

Having established that our direct QCD calculation reproduces the leading-power result of the SCET factorization theorem, we proceed to study the next-toleading-power (NLP) using the same approach. There are three sources of corrections to consider: subleading power terms in the phase space expression of Eq. (34), the subleading power correction in the matrix element in Eq. (40), and the expansion of the soft-region integral of Eq. (46) to subleading order in $1-z_{a}$. The contributions from the first two pieces can be divided into beam and soft regions, following the split of the $z_{a}$ integral performed for the leading-power term in Eq. (42). The beam-region contribution to the NLP result takes the form 


$$
\begin{aligned}
\frac{\mathrm{d} \sigma_{\text {beam }}^{\mathrm{NLP},(a)}}{\mathrm{d} \mathcal{T}}= & \left(\frac{C_{A} \alpha_{s}}{\pi}\right) \int_{0}^{1} \mathrm{~d} x_{a} \int_{0}^{1} \mathrm{~d} x_{b} \frac{(2 \pi) f_{g}\left(x_{b}\right)}{2 s x_{a} x_{b}} \delta\left(s x_{a} x_{b}-m_{H}^{2}\right)|\mathcal{M}(g g \rightarrow H)|^{2} \\
& \times \frac{Q_{a}}{m_{H}^{2}} \int_{x_{a}}^{1} \frac{\mathrm{d} z_{a}}{z_{a}}\left\{\frac{\delta\left(1-z_{a}\right)}{\varepsilon}\left[\left(1-x_{a} \frac{Q_{a}^{\prime}}{Q_{a}}\right) f_{g}\left(x_{a}\right)-x_{a} f_{g}^{\prime}\left(x_{a}\right)\right]\right. \\
& +\delta\left(1-z_{a}\right) \log \left(\frac{Q_{a} \mathcal{T}}{\mu^{2}}\right)\left[\left(-1+\frac{Q_{a}^{\prime}}{Q_{a}} x_{a}\right) f_{g}\left(x_{a}\right)+x_{a} f_{g}^{\prime}\left(x_{a}\right)\right] \\
& -f_{g}\left(\frac{x_{a}}{z_{a}}\right) \mathcal{L}_{0}\left(1-z_{a}\right)+f_{g}\left(x_{a}\right) \delta\left(1-z_{a}\right)+f_{g}\left(\frac{x_{a}}{z_{a}}\right)\left[3+\frac{1}{z_{a}^{2}}-\frac{1}{z_{a}}+z_{a}\right] \\
& \left.+x_{a} \frac{\left(1-z_{a}+z_{a}^{2}\right)^{2}}{z_{a}^{3}} \mathcal{L}_{0}\left(1-z_{a}\right)\left[f_{g}^{\prime}\left(\frac{x_{a}}{z_{a}}\right)+\frac{Q_{a}^{\prime}}{Q_{a}} z_{a} f_{g}\left(\frac{x_{a}}{z_{a}}\right)\right]\right\} .
\end{aligned}
$$

For the soft region we also must include the contribution from the subleading term in the $1-z_{a}$ expansion of Eq. (46), leading to the total soft contribution

$$
\begin{aligned}
\frac{\mathrm{d} \sigma_{\mathrm{soft}}^{\mathrm{NLP},(a)}}{\mathrm{d} \mathcal{T}}= & \left(\frac{C_{A} \alpha_{s}}{\pi}\right) \int_{0}^{1} \mathrm{~d} x_{a} \int_{0}^{1} \mathrm{~d} x_{b} \frac{(2 \pi) f_{g}\left(x_{b}\right)}{2 s x_{a} x_{b}} \delta\left(s x_{a} x_{b}-m_{H}^{2}\right)|\mathcal{M}(g g \rightarrow H)|^{2} \\
& \times \frac{Q_{a}}{m_{H}^{2}}\left\{-\frac{1}{\varepsilon}\left[\left(1-\frac{Q_{a}^{\prime} x_{a}}{Q_{a}}\right) f_{g}\left(x_{a}\right)-x_{a} f_{g}^{\prime}\left(x_{a}\right)\right]-f_{g}\left(x_{a}\right)\right. \\
& \left.+\log \left(\frac{\mathcal{T}^{2}}{\mu^{2}}\right)\left[\left(1-\frac{Q_{a}^{\prime} x_{a}}{Q_{a}}\right) f_{g}\left(x_{a}\right)-x_{a} f_{g}^{\prime}\left(x_{a}\right)\right]-\left(\frac{Q_{b}}{Q_{a}}\right) x_{a} f_{g}^{\prime}\left(x_{a}\right)\right\} .
\end{aligned}
$$

The full NLP correction when the emitted gluon is close to the $n^{\mu}$ direction can be found by simply summing the beam and soft contribution. As expected, the apparent pole cancels:

$$
\begin{aligned}
\frac{\mathrm{d} \sigma^{\mathrm{NLP},(a)}}{\mathrm{d} \mathcal{T}}= & \left(\frac{C_{A} \alpha_{s}}{\pi}\right) \int_{0}^{1} \mathrm{~d} x_{a} \int_{0}^{1} \mathrm{~d} x_{b} \frac{(2 \pi) f_{g}\left(x_{b}\right)}{2 s x_{a} x_{b}} \delta\left(s x_{a} x_{b}-m_{H}^{2}\right)|\mathcal{M}(g g \rightarrow H)|^{2} \\
& \times \frac{Q_{a}}{m_{H}^{2}} \int_{x_{a}}^{1} \frac{\mathrm{d} z_{a}}{z_{a}}\left\{-f_{g}\left(\frac{x_{a}}{z_{a}}\right) \mathcal{L}_{0}\left(1-z_{a}\right)+f_{g}\left(\frac{x_{a}}{z_{a}}\right)\left[3+\frac{1}{z_{a}^{2}}-\frac{1}{z_{a}}+z_{a}\right]\right. \\
& +x_{a} \frac{\left(1-z_{a}+z_{a}^{2}\right)^{2}}{z_{a}^{3}} \mathcal{L}_{0}\left(1-z_{a}\right)\left[f_{g}^{\prime}\left(\frac{x_{a}}{z_{a}}\right)+\frac{Q_{a}^{\prime}}{Q_{a}} z_{a} f_{g}\left(\frac{x_{a}}{z_{a}}\right)\right] \\
& \left.-\delta\left(1-z_{a}\right)\left(\frac{Q_{b}}{Q_{a}}\right) x_{a} f_{g}^{\prime}\left(x_{a}\right)+\delta\left(1-z_{a}\right) \log \left(\frac{\mathcal{T}}{Q_{a}}\right)\left[\left(1-\frac{Q_{a}^{\prime} x_{a}}{Q_{a}}\right) f_{g}\left(x_{a}\right)-x_{a} f_{g}^{\prime}\left(x_{a}\right)\right]\right\} .
\end{aligned}
$$

The total differential cross section is the sum of the two regions $(a)$ and $(b)$ :

$$
\frac{\mathrm{d} \sigma^{\mathrm{NLP}}}{\mathrm{d} \mathcal{T}}=\frac{\mathrm{d} \sigma^{\mathrm{NLP},(a)}}{\mathrm{d} \mathcal{T}}+\{a \leftrightarrow b\}
$$

This is the full $\mathcal{O}(1)$ power correction for the $g g$ channel, including both the LL and NLL terms. Results for the other channels are given in the Appendix.

Let us first focus on the LL $\log \mathcal{T}$ term. Combining both regions we arrive at the following form valid for arbitrary $Q_{a}$ and $Q_{b}$, subject to the (easily removeable) restriction that $Q_{a} Q_{b}=m_{H}^{2}$ :

$$
\begin{aligned}
\frac{\mathrm{d} \sigma_{\mathrm{LL}}^{\mathrm{NLP}}}{\mathrm{d} \mathcal{T}}= & \left(\frac{C_{A} \alpha_{s}}{\pi}\right) \int_{0}^{1} \mathrm{~d} x_{a} \int_{0}^{1} \mathrm{~d} x_{b} \frac{(2 \pi)}{2 s x_{a} x_{b}} \delta\left(s x_{a} x_{b}-m_{H}^{2}\right)|\mathcal{M}(g g \rightarrow H)|^{2} \\
& \times\left\{\frac{Q_{a}}{m_{H}^{2}} \log \left(\frac{\mathcal{T}}{Q_{a}}\right) f_{g}\left(x_{b}\right)\left[\left(1-\frac{Q_{a}^{\prime} x_{a}}{Q_{a}}\right) f_{g}\left(x_{a}\right)-x_{a} f_{g}^{\prime}\left(x_{a}\right)\right]\right. \\
& \left.+\frac{Q_{b}}{m_{H}^{2}} \log \left(\frac{\mathcal{T}}{Q_{b}}\right) f_{g}\left(x_{a}\right)\left[\left(1-\frac{Q_{b}^{\prime} x_{b}}{Q_{b}}\right) f_{g}\left(x_{b}\right)-x_{b} f_{g}^{\prime}\left(x_{b}\right)\right]\right\} .
\end{aligned}
$$

We can apply this form to obtain the LL power corrections to the leptonic and hadronic definitions of $\mathcal{T}$ introduced in Sec. II. For the leptonic $\mathcal{T}$ definition we have 


$$
Q_{a}=Q_{b}=m_{H} \quad Q_{a}^{\prime}=Q_{b}^{\prime}=0,
$$

and therefore the leading-logarithmic power corrections are

$$
\begin{aligned}
\frac{\mathrm{d} \sigma_{\mathrm{LL}}^{\mathrm{NLP}}}{\mathrm{d} \mathcal{T}}= & \left(\frac{C_{A} \alpha_{s}}{\pi}\right) \int_{0}^{1} \mathrm{~d} x_{a} \int_{0}^{1} \mathrm{~d} x_{b} \frac{(2 \pi)}{2 s x_{a} x_{b}} \delta\left(s x_{a} x_{b}-m_{H}^{2}\right)|\mathcal{M}(g g \rightarrow H)|^{2} \\
& \times\left\{\frac{1}{m_{H}} \log \left(\frac{\mathcal{T}}{m_{H}}\right)\left[2 f_{g}\left(x_{a}\right) f_{g}\left(x_{b}\right)-x_{a} f_{g}^{\prime}\left(x_{a}\right) f_{g}\left(x_{b}\right)-x_{b} f_{g}\left(x_{a}\right) f_{g}^{\prime}\left(x_{b}\right)\right]\right\} .
\end{aligned}
$$

This result is consistent with [32]. However, for the hadronic $\mathcal{T}$ we have

$$
Q_{a}=x_{a} \sqrt{s} \quad Q_{b}=x_{b} \sqrt{s} \quad Q_{a}^{\prime}=Q_{b}^{\prime}=\sqrt{s} .
$$

The result becomes

$$
\begin{aligned}
\frac{\mathrm{d} \sigma_{\mathrm{LL}}^{\mathrm{NLP}}}{\mathrm{d} \mathcal{T}}= & \left(\frac{C_{A} \alpha_{s}}{\pi}\right) \int_{0}^{1} \mathrm{~d} x_{a} \int_{0}^{1} \mathrm{~d} x_{b} \frac{(2 \pi)}{2 s x_{a} x_{b}} \delta\left(s x_{a} x_{b}-m_{H}^{2}\right)|\mathcal{M}(g g \rightarrow H)|^{2}\left\{\frac{\sqrt{s} x_{a}}{m_{H}^{2}} \log \left(\frac{\mathcal{T}}{\sqrt{s} x_{a}}\right) f_{g}\left(x_{b}\right)\left[-x_{a} f_{g}^{\prime}\left(x_{a}\right)\right]\right. \\
& \left.+\frac{\sqrt{s} x_{b}}{m_{H}^{2}} \log \left(\frac{\mathcal{T}}{\sqrt{s} x_{b}}\right) f_{g}\left(x_{a}\right)\left[-x_{b} f_{g}^{\prime}\left(x_{b}\right)\right]\right\}
\end{aligned}
$$

We see that the term proportional to $f_{g}\left(x_{a}\right) f_{g}\left(x_{b}\right)$ vanishes when taking into account the derivative of $Q_{a}$ with respect to $x_{a}$. This is consistent with [31] but not with [32]. This result would match that of Ref. [32] if the derivatives of $Q_{i}$ were erroneously neglected in this expression. We suspect that this is the source of difference between the two results. We note that the $Q_{i}$ derivative terms come from the subleading power expansion of the phase space. Since the matrix elements for the quark-gluon channel contribution to Higgs production are already power-suppressed, this partonic process receives contributions only from the leading-power phase space. The $Q_{i}$ derivative terms therefore do not contribute to this channel, and the results of Refs. [31,32] consequently agree. We will also provide in the next section numerical evidence suggesting that Eq. (62) is the correct LL power correction for the hadronic $\mathcal{T}$ definition.

\section{NUMERICAL RESULTS}

We study in this section the numerical impact of the power corrections computed in the previous section. Our intent is to compare the full NLL power corrections with the LL ones, the hadronic $\mathcal{T}$ definition versus the leptonic definition, and the hadronic $\mathcal{T}$ found here versus the result of Ref. [32]. We first consider the leading gluon-gluon partonic channel, before also considering the $q g+g q$ and $q \bar{q}$ results. The following parameter choices are used in all results:

$$
\sqrt{s}=13 \mathrm{TeV}, \quad \mu_{R}=\mu_{F}=m_{H}=125 \mathrm{GeV}
$$

All numerical results are obtained using NNPDF 3.0 NNLO parton distribution functions [40] and MCFM 8.0 [41]. We expand the cross section in the strong coupling constant according to

$$
\sigma_{\mathrm{tot}}=\sigma_{\mathrm{LO}}+\sigma_{\mathrm{NLO}}+\ldots
$$

and display results for the NLO coefficient $\sigma_{\mathrm{NLO}}$ below.

The hadronic and leptonic $\mathcal{T}$ definitions differ in their rapidity dependence, as pointed out in Ref. [30]. We therefore study two observables when comparing the various power corrections: the inclusive cross section and the cross section differential in Higgs rapidity for the forward region $\left|Y_{H}\right|=3$. Finally, we note that the leading-logarithmic power corrections in Eq. (58) are defined differentially in $\mathcal{T}$. When the below-cut contribution which includes this power correction is integrated up to $\mathcal{T}^{\text {cut }}$ to obtain the cross section using $N$-jettiness subtraction, both leading-logarithmic and subleading power corrections in $\mathcal{T}^{\text {cut }}$ are produced:

$$
\int_{0}^{\mathcal{T}^{\text {cut }}} \mathrm{d} \mathcal{T} \log \left(\frac{\mathcal{T}}{Q}\right)=\mathcal{T}^{\text {cut }}\left[\log \left(\frac{\mathcal{T}^{\text {cut }}}{Q}\right)-1\right] .
$$

In our definition of the leading-logarithmic power corrections for the cross section integrated in $\mathcal{T}$ we include both terms arising from the integral above.

We begin by comparing the impact of the power corrections on the hadronic $\mathcal{T}$ distribution. Shown in Fig. 1 are the deviations of the NLO coefficient obtained using $N$-jettiness subtraction as a function of $\mathcal{T}^{\text {cut }}$ from the dipole-subtraction result for three different cases: no power 

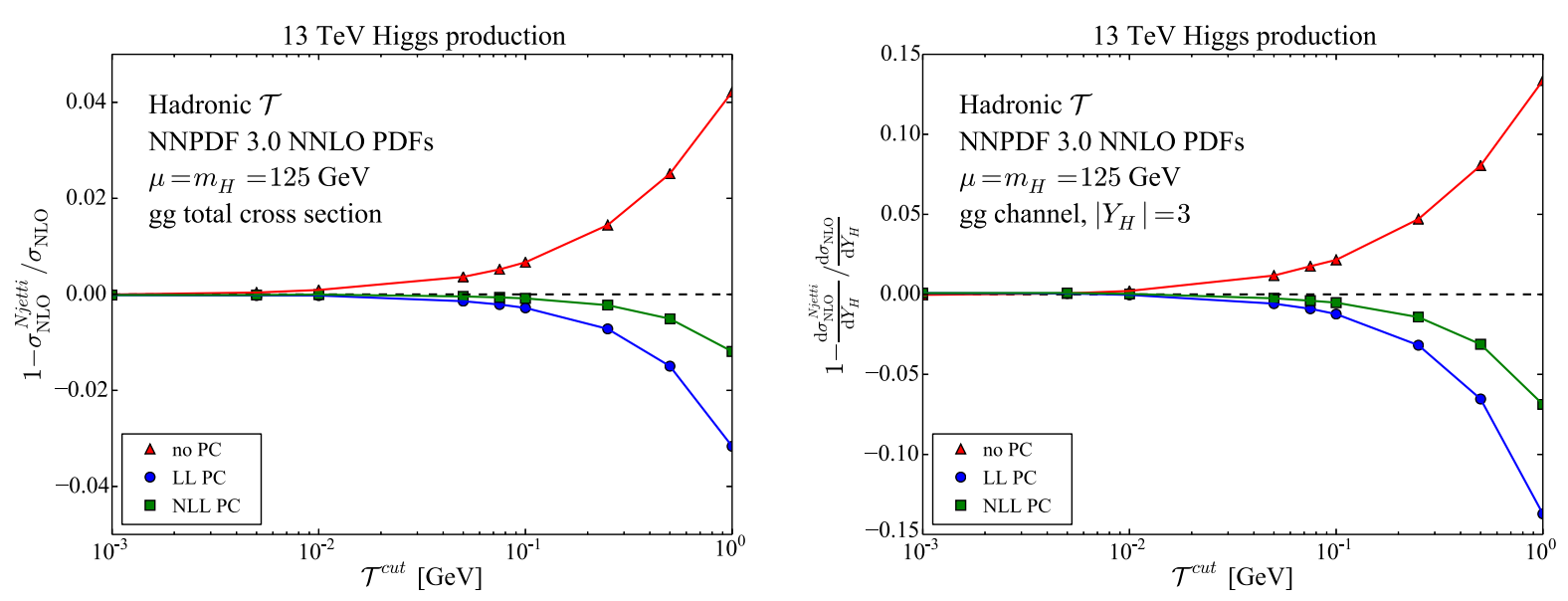

FIG. 1. Deviations of the NLO coefficient obtained using $N$-jettiness subtraction as a function of $\mathcal{T}^{\text {cut }}$ from the dipole-subtraction result for three different cases: no power corrections included, only the leading-logarithmic (LL) power corrections included, and the full NLL power corrections included.

corrections included, only the LL power corrections of Eq. (58) included, and the full NLL power corrections of Eqs. (56) and (57) included. Both the total cross section and the rapidity spectrum for the forward value $\left|Y_{H}\right|=3$ are shown. We observe a substantial improvement as first the LL and then the NLL power corrections are added to the leading-power SCET factorization theorem. The deviation of the NLO coefficient without power corrections reaches over $4 \%$ at $\mathcal{T}^{\text {cut }}=1 \mathrm{GeV}$. This is reduced to $3 \%$ with the LL improvements, and to $1 \%$ at NLL. Requiring a deviation from the exact result of less than $1 \%$ requires $\mathcal{T}^{\text {cut }} \leq 0.1 \mathrm{GeV}$ without power corrections. This is reduced to $\mathcal{T}^{\text {cut }} \leq 0.25 \mathrm{GeV}$ with LL power corrections included and further to $\mathcal{T}^{\text {cut }} \leq 1 \mathrm{GeV}$ with both $\mathrm{LL}$ and NLL corrections included. The pattern of improvement as the power corrections are added is similar at $\left|Y_{H}\right|=3$, except with larger deviations due to the nature of the hadronic $\mathcal{T}$ definition. Incorporating the NLL power corrections reduces the deviation from the dipole subtraction result

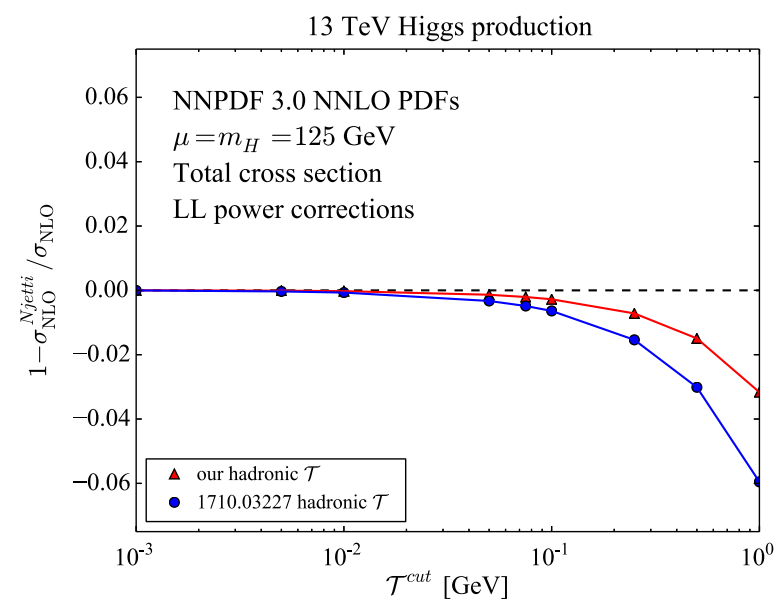

for the $\left|Y_{H}\right|=3$ region by a factor of 2 compared to having just the LL power correction.

Before proceeding to study the leptonic $\mathcal{T}$ definition we first discuss the numerical difference between the LL power correction results of Refs. [31,32] for the hadronic case. We can obtain the result of Ref. [32] by neglecting the $Q_{i}$ derivatives in Eq. (58), as discussed in the previous section. We again show the deviations for the total cross section and the differential one for forward rapidity as a function of $\mathcal{T}^{\text {cut }}$ in Fig. 2. For both the total cross section and the forward-rapidity region the deviations from the result of dipole subtraction are nearly a factor of two larger for the Ref. [32] result than for our expression, providing numerical evidence for the result obtained here and in Ref. [31].

We now study the impact of power corrections on the leptonic $\mathcal{T}$ variable. We also compare the deviations from the dipole subtraction result between the hadronic and leptonic definitions. We first show in Fig. 3 the comparison of the two $\mathcal{T}$ definitions without power corrections for the

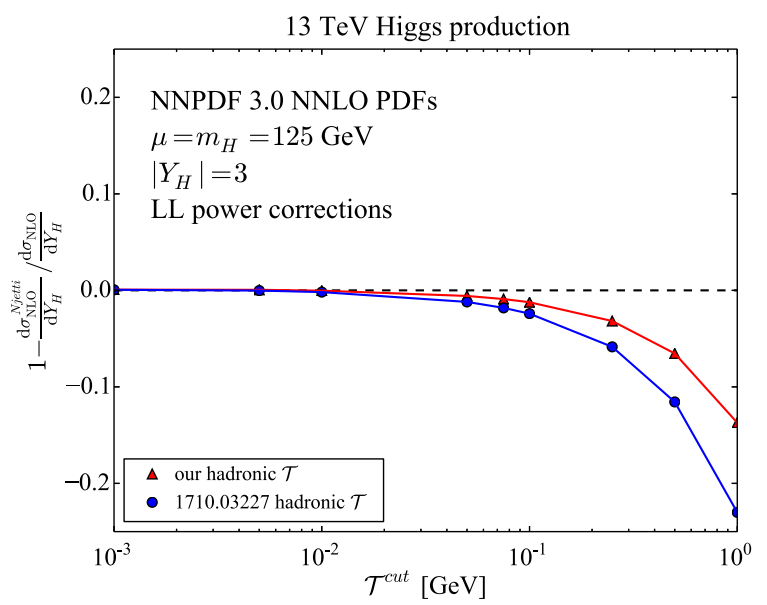

FIG. 2. Study of the differing definitions of the LL power corrections in the hadronic $\mathcal{T}$ case between this work and Ref. [32] (labeled 1710.03227 according to its arXiv number). 

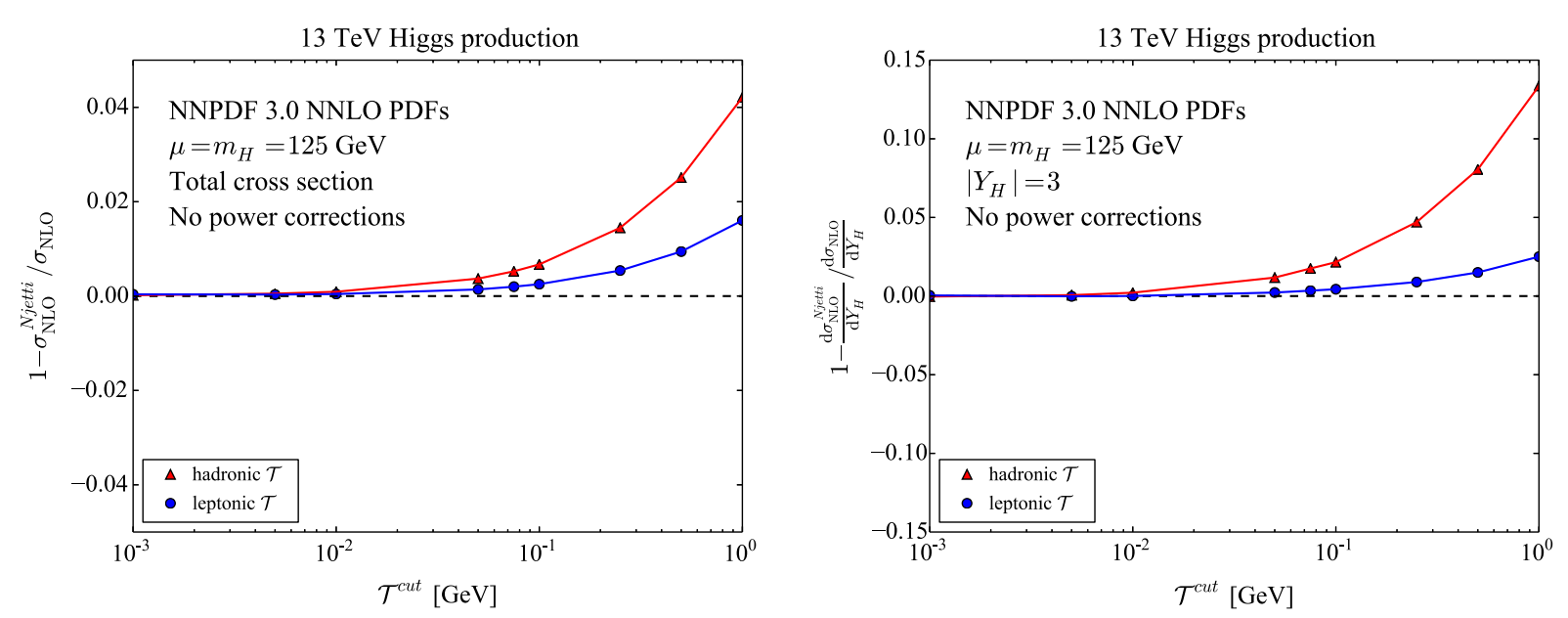

FIG. 3. Comparison of the leptonic and hadronic $\mathcal{T}$ deviations from dipole subtraction for the total cross section (left panel) and forward rapidity region (right panel). No power corrections are included.

total cross section and the forward rapidity region. The leptonic definition exhibits less deviation from the dipole subtraction result for both observables, but it is especially better behaved for the forward rapidity region, where it exhibits only a $2 \%$ discrepancy even for $\mathcal{T}^{\text {cut }}=1 \mathrm{GeV}$. Both definitions are better behaved when the NLL power corrections are included, as shown in Fig. 4. In particular the leptonic definition becomes nearly identical to the dipole subtraction result up to $\mathcal{T}^{\text {cut }}=1 \mathrm{GeV}$. This has a significant effect on the numerical efficiency of the method. Each of the above-cut and below-cut contributions depends separately on $\mathcal{T}^{\text {cut }}$ as $\log ^{2}\left(\mathcal{T}^{\text {cut }}\right)$, and these terms only cancel after the two pieces are added. We note that with a fixed number of integrand evaluations for our numerical integration, the estimated statistical error is a factor of 2 smaller for $\mathcal{T}^{\text {cut }}=1 \mathrm{GeV}$ than for $\mathcal{T}^{\text {cut }}=0.05 \mathrm{GeV}$, which would have to be used for subpercent agreement with the dipole subtraction result for the hadronic definition without power corrections.

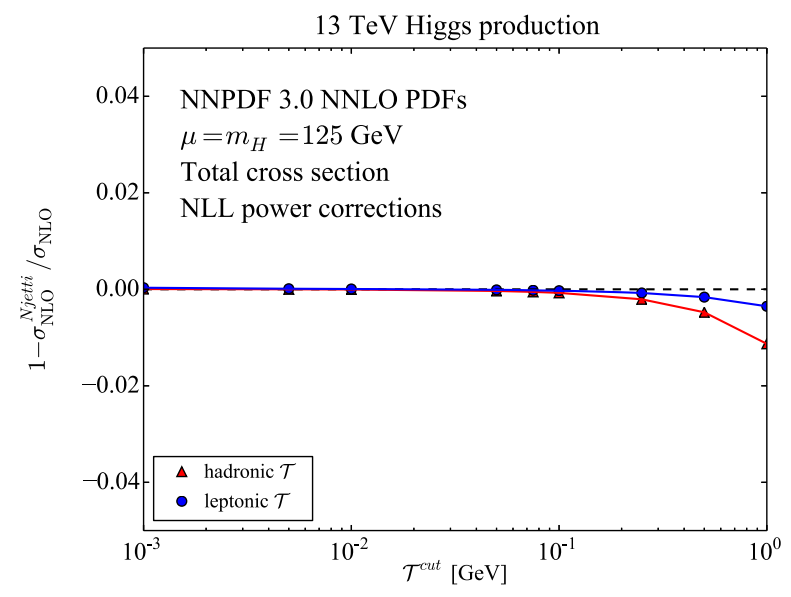

We now consider the $q g+g q$ initial state. Analytic results for this channel are given in the Appendix. We focus on the hadronic $\mathcal{T}$ definition and study the impact of including the full NLL power corrections. We note, however, that our derivation is equally valid for all definitions of $\mathcal{T}$, including for the leptonic one. Results for the inclusive cross section and for the forward rapidity value $\left|Y_{H}\right|=3$ are shown are shown in Fig. 5. It is interesting to note that the LL power corrections worsen the agreement between $N$-jettiness subtraction and the dipole subtraction result for both the total cross section and the forward rapidity region. Only upon including the full NLL power corrections is a better agreement with the dipole subtraction result obtained. We note that for the total cross section the agreement at NLL is excellent, with subpercent deviations observed all the way up to $\mathcal{T}^{\text {cut }}=1 \mathrm{GeV}$. The deviation at LL reaches $20 \%$ at this $\mathcal{T}^{\text {cut }}$ value. This pattern cannot be seen when studying the full NLO cross section since the $q g$ channel is much smaller than the dominant $g g$ scattering

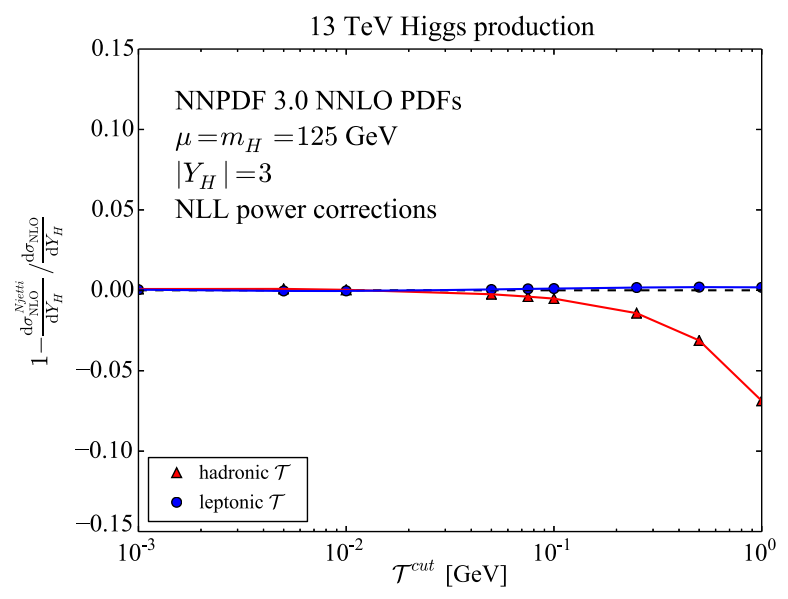

FIG. 4. Comparison of the leptonic and hadronic $\mathcal{T}$ deviations from dipole subtraction for the total cross section (left panel) and forward rapidity region (right panel). The full $\mathcal{O}(\mathcal{T})$ power corrections are included. 

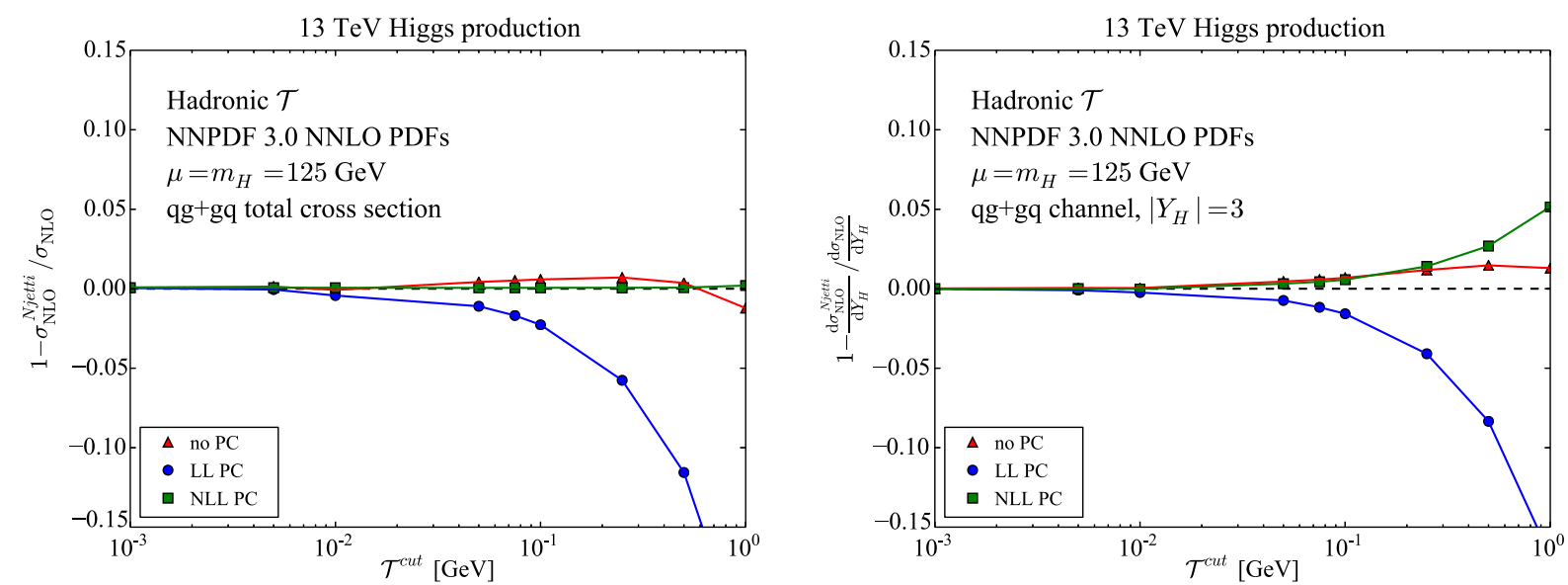

FIG. 5. Deviations of the $q g+g q$ NLO coefficient obtained using $N$-jettiness subtraction as a function of $\mathcal{T}^{\text {cut }}$ from the dipolesubtraction result for three different cases: no power corrections included, only the leading-logarithmic (LL) power corrections included, and the full NLL power corrections included.
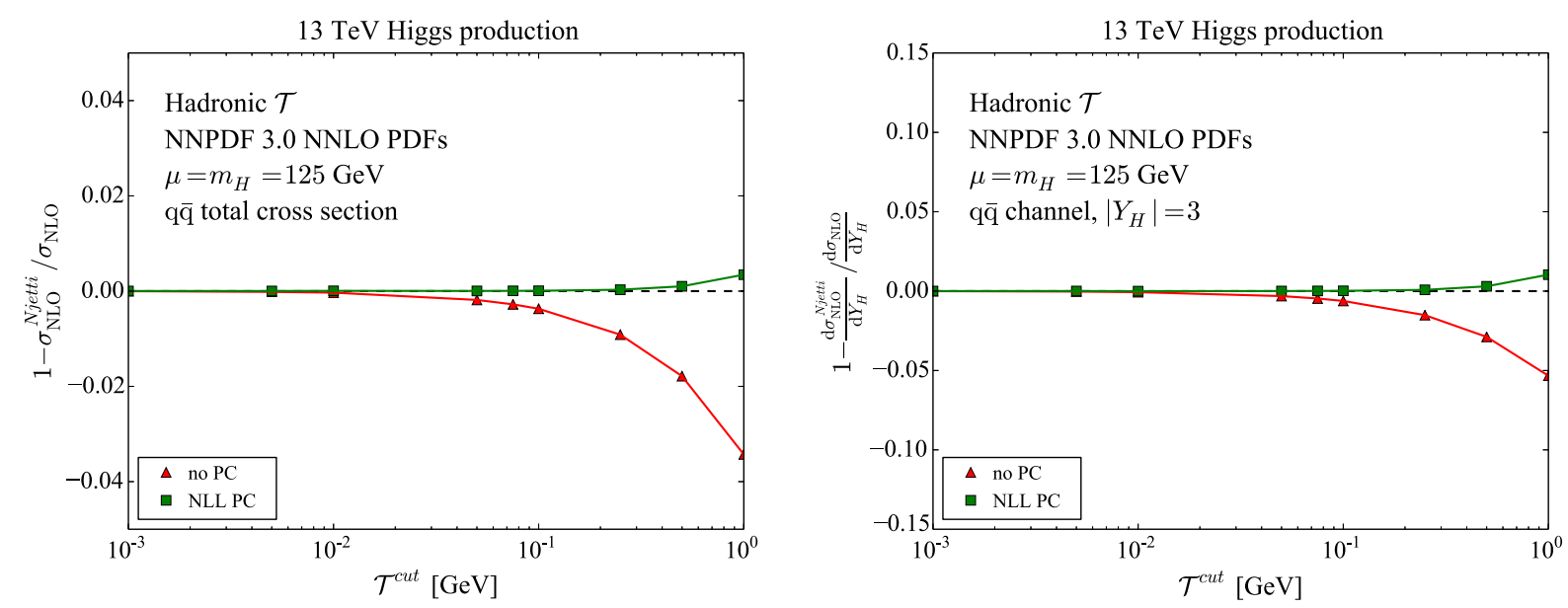

FIG. 6. Deviations of the $q \bar{q}$ NLO coefficient obtained using $N$-jettiness subtraction as a function of $\mathcal{T}^{\text {cut }}$ from the dipole-subtraction result for three different cases: no power corrections included and the full NLL power corrections included.

process. For the forward rapidity region the NLL power corrections deviate from the dipole subtraction more than the result without power corrections at large $\mathcal{T}^{\text {cut }}$. We note that the deviation between the no power-correction result and the dipole subtraction cross section is nonmonotonic as a function of $\mathcal{T}^{\text {cut }}$, indicating an accidental cancellation between subleading powers that leads to the observed behavior. The full NLL corrections still produce a subpercent deviation from the dipole subtraction result up to fairly large values $\mathcal{T}^{\text {cut }} \approx 0.3 \mathrm{GeV}$.

Finally we consider the $q \bar{q}$ initial state. Analytic results for this channel are given in the Appendix. This contribution is numerically very subdominant to the other channels. It does not have either a leading-power or LL subleading power result, and begins first at the NLL level. Results for the inclusive cross section and for the forward rapidity value $\left|Y_{H}\right|=3$ are shown are shown in Fig. 6 .
The agreement between the dipole and $N$-jettiness subtraction results after including the NLL power corrections is excellent for both the total cross section and the forward rapidity region, with subpercent deviations for all studied values of $\mathcal{T}^{\text {cut }}$.

\section{CONCLUSIONS}

In this manuscript we have studied in detail the power corrections to the effective-theory factorization theorem for the 0 -jettiness event shape variable. In comparison to previous works we have derived the next-to-leadinglogarithmic power corrections at next-to-leading order, and not just the leading-logarithmic corrections obtained previously. We have derived our result directly in QCD, without use of the effective field theory formalism, and have discussed the connection between this method and the 
SCET approach. We hope that our discussion is of interest to the reader who desires to better understand the connection between traditional QCD and the effectivetheory approach. We have also addressed a discrepancy between different results for the leading-logarithmic power corrections to the hadronic $\mathcal{T}$ variable that has appeared in the literature. We have shown that the derivation of Refs. [30,32] seems to neglect a contribution appearing in the factorization of the phase space that does not appear for the leptonic $\mathcal{T}$ definition of primary interest there. This additional factor only appears for partonic processes that have leading-power matrix elements, explaining why the corrections for the quark-gluon scattering channels in Refs. [31,32] agree while the gluon-gluon channel does not. We have provided numerical evidence for the correctness of the result of Ref. [31]. We have also presented a numerical study of the power corrections for both the hadronic and leptonic $\mathcal{T}$ definitions. Including the NLL power corrections improves the performance of the $N$-jettiness subtraction method applied to color-singlet production. For the hadronic $\mathcal{T}$, including the power corrections reduces the deviation from the NLO result as computed by dipole subtraction by nearly a factor of three. The leptonic $\mathcal{T}$ shows nearly no deviation from the dipole subtraction result after the power corrections are included. This significantly improves the numerical efficiency of the $N$-jettiness subtraction method.

It would be interesting in the future to extend the derivation here to the subleading logarithmic level at NNLO as well, which we believe is possible. Applications of $N$-jettiness subtraction to jet production would also benefit from an understanding of power corrections, which should benefit from the techniques used in our direct QCD derivation of the 0-jettiness power corrections. We look forward to these future investigations.

\section{ACKNOWLEDGMENTS}

R. B. is supported by the Department of Energy (DOE) under Contract No. DE-AC02-06CH11357. F. P. is supported by the DOE Grants No. DE-FG02-91ER40684 and No. DE-AC02-06CH11357. A. I. is supported by the DOE Grant No. DE-FG02-91ER40684 and the National Science Foundation (NSF) Grant No. NSF-1520916. This research used resources of the Argonne Leadership Computing Facility, which is a DOE Office of Science User Facility supported under Contract No. DE-AC02-06CH11357. R. B. and F.P. thank the Aspen Center for Physics and the Perimeter Institute for kind hospitality during the course of this work. This research was supported in part by Perimeter Institute for Theoretical Physics. Research at Perimeter Institute is supported by the Government of Canada through Industry Canada and by the Province of Ontario through the Ministry of Economics Development and Innovation.

\section{APPENDIX: RESULTS FOR INITIAL-STATE QUARK CHANNELS}

We compile here the results for the numerically smaller partonic channels, focusing on the $n$-collinear sector as before. There are three channels to consider: $q \bar{q} \rightarrow H g$, $q g \rightarrow H q$, and $g q \rightarrow H q$ (when considering only the $n$ collinear sector the last two channels are different). We show the results for each channel in the $n$-collinear sector. The $\bar{n}$-collinear sector is obtained by substituting $a \leftrightarrow b$ (and consequently $g q \leftrightarrow q g$ ). The full result is the sum of the $n$-collinear and $\bar{n}$-collinear sectors.

\section{1. $q \bar{q} \rightarrow H g$}

There is no leading-power contribution from this channel, as the matrix element is subleading in $\mathcal{T}$. There is also no leading-logarithmic power correction. The NLL power correction is

$$
\frac{\mathrm{d} \sigma^{\mathrm{NLP},(a)}}{\mathrm{d} \mathcal{T}}=\frac{4}{3} \frac{\alpha_{s} C_{F}}{\pi} \int_{0}^{1} \mathrm{~d} x_{a} \int_{0}^{1} \mathrm{~d} x_{b} \frac{(2 \pi) f_{\bar{q}}\left(x_{b}\right)}{2 s x_{a} x_{b}} \delta\left(s x_{a} x_{b}-m_{H}^{2}\right)|\mathcal{M}(g g \rightarrow H)|^{2} \times \frac{Q_{a}}{m_{H}^{2}} \int_{x_{a}}^{1} \frac{\mathrm{d} z_{a}}{z_{a}} \frac{\left(1-z_{a}\right)^{2}}{z_{a}} f_{q}\left(\frac{x_{a}}{z_{a}}\right) .
$$

\section{2. $g q \rightarrow H q$}

There is again no leading-power contribution. The next-to-leading-power has a LL and a NLL term:

$$
\begin{aligned}
\frac{\mathrm{d} \sigma^{\mathrm{NLP},(a)}}{\mathrm{d} \mathcal{T}}= & \frac{\alpha_{s} C_{F}}{2 \pi} \int_{0}^{1} \mathrm{~d} x_{a} \int_{0}^{1} \mathrm{~d} x_{b} \frac{(2 \pi) f_{q}\left(x_{b}\right)}{2 s x_{a} x_{b}} \delta\left(s x_{a} x_{b}-m_{H}^{2}\right)|\mathcal{M}(g g \rightarrow H)|^{2} \\
& \times \frac{Q_{a}}{m_{H}^{2}} \int_{x_{a}}^{1} \frac{\mathrm{d} z_{a}}{z_{a}}\left\{-\delta\left(1-z_{a}\right) f_{g}\left(x_{a}\right) \log \left(\frac{\mathcal{T}}{Q_{a}}\right)+\frac{\mathcal{L}_{0}\left(1-z_{a}\right)}{z_{a}} f_{g}\left(\frac{x_{a}}{z_{a}}\right)\right\} .
\end{aligned}
$$

\section{3. $q \mathrm{~g} \rightarrow \mathrm{Hq}$}

There is a nonzero leading-power contribution that comes from the beam function, and that is already in the literature. The next-to-leading-power has no leading-logarithmic contribution, but it does have a nonzero NLL contribution: 


$$
\begin{aligned}
\frac{\mathrm{d} \sigma^{\mathrm{NLP},(a)}}{\mathrm{d} \mathcal{T}}= & \frac{\alpha_{s} C_{F}}{2 \pi} \int_{0}^{1} \mathrm{~d} x_{a} \int_{0}^{1} \mathrm{~d} x_{b} \frac{(2 \pi) f_{g}\left(x_{b}\right)}{2 s x_{a} x_{b}} \delta\left(s x_{a} x_{b}-m_{H}^{2}\right)|\mathcal{M}(g g \rightarrow H)|^{2} \\
& \times \int_{x_{a}}^{1} \frac{\mathrm{d} z_{a}}{z_{a}}\left\{\frac{Q_{a}}{m_{H}^{2}} \frac{2-2 z_{a}+z_{a}^{2}}{z_{a}^{3}}\left[x_{a} f_{q}^{\prime}\left(\frac{x_{a}}{z_{a}}\right)+x_{a} z_{a} \frac{Q_{a}^{\prime}}{Q_{a}} f_{q}\left(\frac{x_{a}}{z_{a}}\right)\right]\right. \\
& \left.+\frac{Q_{a}}{m_{H}^{2}} \frac{2-2 z_{a}+z_{a}^{2}}{z_{a}^{2}} f_{q}\left(\frac{x_{a}}{z_{a}}\right)-\delta\left(1-z_{a}\right) \frac{Q_{b}}{m_{H}^{2}} f_{q}\left(x_{a}\right)\right\} .
\end{aligned}
$$

[1] A. Gehrmann-De Ridder, T. Gehrmann, and E. W. N. Glover, J. High Energy Phys. 09 (2005) 056.

[2] G. Somogyi, Z. Trocsanyi, and V. Del Duca, J. High Energy Phys. 06 (2005) 024.

[3] S. Catani and M. Grazzini, Phys. Rev. Lett. 98, 222002 (2007).

[4] M. Czakon, Phys. Lett. B 693, 259 (2010).

[5] R. Boughezal, K. Melnikov, and F. Petriello, Phys. Rev. D 85, 034025 (2012).

[6] M. Cacciari, F. A. Dreyer, A. Karlberg, G. P. Salam, and G. Zanderighi, Phys. Rev. Lett. 115, 082002 (2015).

[7] R. Boughezal, C. Focke, X. Liu, and F. Petriello, Phys. Rev. Lett. 115, 062002 (2015).

[8] J. Gaunt, M. Stahlhofen, F. J. Tackmann, and J. R. Walsh, J. High Energy Phys. 09 (2015) 058.

[9] I. W. Stewart, F. J. Tackmann, and W. J. Waalewijn, Phys. Rev. Lett. 105, 092002 (2010).

[10] R. Boughezal, J. M. Campbell, R. K. Ellis, C. Focke, W. T. Giele, X. Liu, and F. Petriello, Phys. Rev. Lett. 116, 152001 (2016).

[11] R. Boughezal, X. Liu, and F. Petriello, Phys. Lett. B 760, 6 (2016).

[12] R. Boughezal, X. Liu, and F. Petriello, Phys. Rev. D 94, 113009 (2016).

[13] R. Boughezal, X. Liu, and F. Petriello, Phys. Rev. D 94, 074015 (2016).

[14] R. Boughezal, C. Focke, W. Giele, X. Liu, and F. Petriello, Phys. Lett. B 748, 5 (2015).

[15] G. Abelof, R. Boughezal, X. Liu, and F. Petriello, Phys. Lett. B 763, 52 (2016).

[16] C. W. Bauer, S. Fleming, and M. E. Luke, Phys. Rev. D 63, 014006 (2000).

[17] C. W. Bauer, S. Fleming, D. Pirjol, and I. W. Stewart, Phys. Rev. D 63, 114020 (2001).

[18] C. W. Bauer and I. W. Stewart, Phys. Lett. B 516, 134 (2001).

[19] C. W. Bauer, D. Pirjol, and I. W. Stewart, Phys. Rev. D 65, 054022 (2002).

[20] C. W. Bauer, S. Fleming, D. Pirjol, I. Z. Rothstein, and I. W. Stewart, Phys. Rev. D 66, 014017 (2002).
[21] D. Bonocore, E. Laenen, L. Magnea, L. Vernazza, and C. D. White, Phys. Lett. B 742, 375 (2015).

[22] D. Bonocore, E. Laenen, L. Magnea, S. Melville, L. Vernazza, and C. D. White, J. High Energy Phys. 06 (2015) 008.

[23] D. Bonocore, E. Laenen, L. Magnea, L. Vernazza, and C. D. White, J. High Energy Phys. 12 (2016) 121.

[24] I. Moult, I. W. Stewart, and G. Vita, J. High Energy Phys. 07 (2017) 067.

[25] I. Feige, D. W. Kolodrubetz, I. Moult, and I. W. Stewart, J. High Energy Phys. 11 (2017) 142.

[26] T. Liu, A. A. Penin, and N. Zerf, Phys. Lett. B 771, 492 (2017).

[27] T. Liu and A. A. Penin, Phys. Rev. Lett. 119, 262001 (2017).

[28] C. H. Chang, I. W. Stewart, and G. Vita, arXiv:1712.04343.

[29] M. Beneke, M. Garny, R. Szafron, and J. Wang, J. High Energy Phys. 03 (2018) 001.

[30] I. Moult, L. Rothen, I. W. Stewart, F. J. Tackmann, and H. X. Zhu, Phys. Rev. D 95, 074023 (2017).

[31] R. Boughezal, X. Liu, and F. Petriello, J. High Energy Phys. 03 (2017) 160.

[32] I. Moult, L. Rothen, I. W. Stewart, F. J. Tackmann, and H. X. Zhu, Phys. Rev. D 97, 014013 (2018).

[33] C. F. Berger, C. Marcantonini, I. W. Stewart, F. J. Tackmann, and W. J. Waalewijn, J. High Energy Phys. 04 (2011) 092.

[34] J. Gaunt, M. Stahlhofen, and F. J. Tackmann, J. High Energy Phys. 08 (2014) 020.

[35] T. T. Jouttenus, I. W. Stewart, F. J. Tackmann, and W. J. Waalewijn, Phys. Rev. D 83, 114030 (2011).

[36] S. Dawson, Nucl. Phys. B359, 283 (1991).

[37] A. Djouadi, M. Spira, and P. M. Zerwas, Phys. Lett. B 264, 440 (1991).

[38] V. Ahrens, T. Becher, M. Neubert, and L. L. Yang, Eur. Phys. J. C 62, 333 (2009).

[39] R. V. Harlander, Phys. Lett. B 492, 74 (2000).

[40] R. D. Ball et al. (NNPDF Collaboration), J. High Energy Phys. 04 (2015) 040.

[41] R. Boughezal, J. M. Campbell, R. K. Ellis, C. Focke, W. Giele, X. Liu, F. Petriello, and C. Williams, Eur. Phys. J. C 77, 7 (2017). 